\title{
Subscales on the element boundaries in the variational two-scale finite element method
}

\author{
Ramon Codina*, Javier Principe, Joan Baiges \\ Universitat Politècnica de Catalunya, Jordi Girona 1-3, Edifici C1, 08034 Barcelona, Spain
}

\section{A R T I C L E I N F O}

\section{Article history:}

Received 14 March 2008

Received in revised form 16 October 2008

Accepted 23 October 2008

Available online 20 November 2008

\section{Keywords:}

Stabilized finite element methods

Stokes

Darcy

Transmission conditions

\begin{abstract}
A B S T R A C T
In this paper, we introduce a way to approximate the subscales on the boundaries of the elements in a variational two-scale finite element approximation to flow problems. The key idea is that the subscales on the element boundaries must be such that the transmission conditions for the unknown, split as its finite element contribution and the subscale, hold. In particular, we consider the scalar convection-diffusion-reaction equation, the Stokes problem and Darcy's problem. For these problems the transmission conditions are the continuity of the unknown and its fluxes through element boundaries. The former is automatically achieved by introducing a single valued subscale on the boundaries (for the conforming approximations we consider), whereas the latter provides the effective condition for approximating these values. The final result is that the subscale on the interelement boundaries must be proportional to the jump of the flux of the finite element component and the average of the subscale calculated in the element interiors.
\end{abstract}

(c) 2008 Elsevier B.V. All rights reserved.

\section{Introduction}

The variational multiscale (VMS) framework to approximate boundary value problems starts with the variational formulation of the problem. In particular, in the two-scale version we consider, it consists in splitting the unknown and the test function into a component in a discrete approximating space and another component in its complement, for which an approximation needs to be proposed. This component is called subgrid scale or, simply, subscale. This idea was proposed in the finite element context in $[16,17]$. The standard Galerkin method accommodates this framework simply by considering the subscales to be negligible.

The main interest of the VMS framework is to develop stabilized finite element methods in a broad sense, meaning that it allows to design discrete variational formulations that do not suffer from the stability problems of the standard Galerkin method. In particular, we are interested here in finite element methods for some model problems arising in fluids mechanics (see [8] for a review of different stabilization methods in flow problems).

The VMS concept as described above is quite general. The way to approximate the subscales is left open. Many questions arise, such as the space for these subscales, the problem to be solved to compute them or their behavior in time dependent problems. In principle, the problem for the subscales is global, that is to say, defined over all the computational domain. In order to simplify

\footnotetext{
* Corresponding author.

E-mail addresses: ramon.codina@upc.edu (R. Codina),principe@cimne.upc.edu (J. Principe), jbaiges@cimne.upc.edu (J. Baiges)
}

it, some sort of localization is necessary, for example by assuming that the subscales vanish on the interelement boundaries, that is to say, they are bubble functions (see for example [4,24] for application of this concept to flow problems).

The treatment of the subscales on the interelement boundaries is precisely the subject of this paper. We propose a way to compute them based on the following ideas:

- We assume the subscales on the element interiors computed and thus the localization process mentioned consists in computing these subscales without accounting for their boundary values.

- The subscales on the element boundaries are single valued, even if they are discontinuous in the element interiors. This requires a hybrid-type formalism to write the exact variational equations that we develop only in the first problem analyzed.

- The subscales on the element boundaries are computed by imposing that the correct transmission conditions of the problem at hand hold. Obviously, these transmission conditions are problem-dependent.

- The fluxes of the subscales on the interelement boundaries are approximated using a simple finite-difference scheme. This is the only approximation we use, apart from those shared with VMS methods that are required to approximate the subscales in the element interiors.

A completely different approach to compute subscales on the interelement boundaries is proposed in [1], where local problems along these boundaries are set. 
We will not insist on other aspects of the VMS method, such as the problem for the subscales in the element interiors, the space where they belong or their time dependency. Let us only mention that we approximate them using an approximate Fourier analysis, that very often we compute them as $L^{2}$-orthogonal to the finite element space [9] and that we consider them time dependent in transient problems $[10,12]$. In order to skip as much as possible this discussion, we will present our formulation without using the explicit expression for the subscales in the element interiors. This approach is, as far as we know, original, and we use it mainly to focus the attention in the expression of the subscales on the interelement boundaries.

The particular transmission conditions between interelement boundaries, that serve us to compute the subscales on these boundaries, are problem dependent. This is why we will treat different problems arising in fluid mechanics, all of them linear and stationary. The first is the convection-diffusion-reaction (CDR) equation considered in Section 2. We show that the subscale on the element boundaries is proportional to the jump of the flux with a negative sign, and also to the average of the subscales computed in the element interiors adjacent to an edge and extended to this edge. In the following, "edge" will be used also in 3D problems, in this case meaning a face. The sign of the subscales on the edges subtracts stability to the problem. However, we show that it is possible to control the new terms added. Neither for this problem nor for the other two discussed in the paper we analyze convergence, since it depends on the particular expression of the subscale on

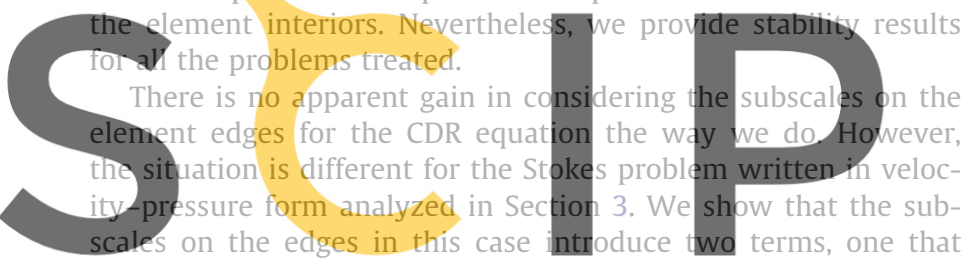
depends on the velocity gradients and that needs to be controlled with the viscous term and another one that provides pressure stabil

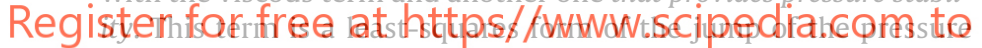
across the edges, and therefore acts only when discontinuous pressure interpolations are used (note that it is not related to the jump stabilization technique proposed for example in [7,6]). The term we add is similar to the one already introduced in [18], which has the local variant proposed and analyzed in $[25,20]$ for the $Q_{1} / P_{0}$ (bilinear-constant) and $P_{1} / P_{0}$ (linear-constant) velocitypressure pairs.

Section 4 describes the application of our ideas to Darcy's problem. We propose a stabilized formulation that includes, with minor modifications, the methods proposed for example in [21] (and extended in [22]) and in [19]. As in the previous cases, we provide a stability result. In this case, the bilinear form associated to the problem is not coercive, but only an inf-sup condition can be proved.

Let us mention that the ideas presented here can be applied to other problems. In particular, in [11] a method to compute the subscales on the element boundaries for the stress-velocity-pressure formulation of the Stokes problem is proposed and fully analyzed. In this case, subscales on the boundaries are essential to deal with discontinuous pressure and stress interpolations.

The main contributions of our approach can be summarized as follows:

- To provide a consistent VMS justification to some stabilizing terms introduced in previous works to deal with discontinuous pressures.

- To propose a symmetric stabilized problem for the Stokes and the Darcy equations (if subscales in the element interiors can be considered negligible compared to the jump of the stresses, see Remark 5). The sign of the symmetric operator, which subtracts stability from the Galerkin terms, is crucial to achieve this symmetry. The situation is similar to what happens when minus the adjoint of the differential operator applied to the test functions is used instead of the original differential operator in the stabilizing terms. This suggestion was first introduced in [14] and turns out to be completely natural in the VMS framework. Also in this case, the diffusive term subtracts stability in the case of the CDR equation.

- Even though we do not exploit this point in this paper, our approach suggests how to stabilize Neumann boundary conditions, essential for example in some fluid-structure interaction problems (see Remark 6).

Some numerical examples are presented in Section 5. Since the stabilizing effect of the boundary terms introduced for the different problems is well known, we simply check what is particular of our approach, namely, the terms that may deteriorate stability. We show that this is not the case in two cases, namely, a convection-diffusion example and two Stokes problems. As the stability analysis dictates, these terms can be controlled by the rest of the terms appearing in the stabilized formulation. Moreover, in the Stokes problem case, some discontinuous pressure interpolations unstable using the Galerkin method, such as the $P_{1} / P_{0}$ pair (see Section 5) can be used.

Finally, some conclusions close the paper in Section 6.

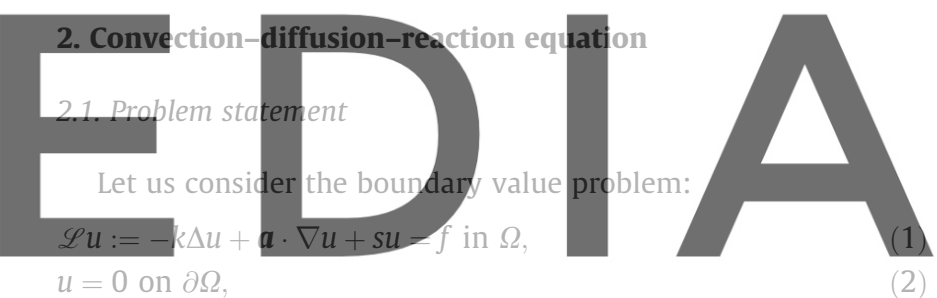

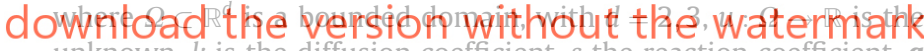
unknown, $k$ is the diffusion coefficient, $s$ the reaction coefficient, $\boldsymbol{a}$ the advection velocity and $f$ the given source term. For simplicity, we assume $k>0, s \geqslant 0$ and the advection velocity $\boldsymbol{a}$ all constants.

Let $V=H_{0}^{1}(\Omega)$ and assume $f \in H^{-1}(\Omega)$. The variational form of the problem consists of finding $u \in V$ such that

$B(u, v):=k(\nabla u, \nabla v)+(\boldsymbol{a} \cdot \nabla u, v)+s(u, v)=\langle f, v\rangle=: L(v) \quad \forall v \in V$

Here and below, $(\cdot, \cdot)$ denotes the $L^{2}$ product in $\Omega$. In general, the integral of two function $g_{1}$ and $g_{2}$ over a domain $\omega$ will be denoted by $\left\langle g_{1}, g_{2}\right\rangle_{\omega}$ and the norm in a function space $X$ by $\|\cdot\|_{X}$, with the simplifications $\|\cdot\|_{L^{2}(\Omega)} \equiv\|\cdot\|$ and $\langle\cdot, \cdot\rangle_{\Omega} \equiv\langle\cdot, \cdot\rangle$. This symbol will also be used for the duality pairing.

\subsection{Six and four field formulations}

As mentioned earlier, we consider that the subscales on the element boundaries are single valued, even if they are discontinuous in the element interiors. To give a variational foundation to the approximation presented in the next subsection, let us consider a hybrid-type approach, starting with a particular six field formulation of the problem. For simplicity, let us assume that $\bar{\Omega}=\bar{\Omega}_{1} \cup \bar{\Omega}_{2}$, with $\Gamma=\partial \Omega_{1} \cap \partial \Omega_{2}$. Consider a decomposition of $V=H_{0}^{1}(\Omega)$ of the form $V=\bar{V} \oplus V^{\prime}$, and let $u=\bar{u}+u^{\prime}$ be the corresponding decomposition of the unknown. Let us state a variational formulation of the problem taking as unknowns $\bar{u}, u^{\prime}$, their traces on $\Gamma$, denoted by $\bar{\gamma}$ and $\gamma^{\prime}$, respectively, and their fluxes, denoted by $\bar{\lambda}$ and $\lambda^{\prime}$, respectively. The space of traces $T=H_{00}^{1 / 2}(\Gamma)$ and the space of fluxes $F=\left(H_{00}^{1 / 2}(\Gamma)\right)^{\prime}$ (the dual of $T$ ) are also assumed to be split as 
$T=T \oplus T^{\prime}$ and $F=F \oplus F^{\prime}$. Note that the prime in $V^{\prime}, T^{\prime}$ and $F^{\prime}$ is not used to denote the dual of a space.

If we denote with a subscript $i$ the restriction of $\bar{u}, u^{\prime}, \bar{\lambda}, \lambda^{\prime}, B$ and $L$ to subdomain $i(i=1,2)$, the problem for the six fields $\bar{u}, u^{\prime}, \bar{\gamma}, \gamma^{\prime}, \bar{\lambda}$ and $\lambda^{\prime}$ can be written as

$B_{1}\left(\bar{u}_{1}, \bar{v}_{1}\right)+B_{1}\left(u_{1}^{\prime}, \bar{v}_{1}\right)-\left\langle\bar{\lambda}_{1}+\lambda_{1}^{\prime}, \bar{v}_{1}\right\rangle_{\Gamma}=L_{1}\left(\bar{v}_{1}\right) \quad \forall \bar{v}_{1}$,

$B_{1}\left(\bar{u}_{1}, v_{1}^{\prime}\right)+B_{1}\left(u_{1}^{\prime}, v_{1}^{\prime}\right)-\left\langle\bar{\lambda}_{1}+\lambda_{1}^{\prime}, v_{1}^{\prime}\right\rangle_{\Gamma}=L_{1}\left(v_{1}^{\prime}\right) \quad \forall v_{1}^{\prime}$,

$B_{2}\left(\bar{u}_{2}, \bar{v}_{2}\right)+B_{2}\left(u_{2}^{\prime}, \bar{v}_{2}\right)-\left\langle\bar{\lambda}_{2}+\lambda_{2}^{\prime}, \bar{v}_{2}\right\rangle_{\Gamma}=L_{2}\left(\bar{v}_{2}\right) \quad \forall \bar{v}_{2}$,

$B_{2}\left(\bar{u}_{2}, v_{2}^{\prime}\right)+B_{2}\left(u_{2}^{\prime}, v_{2}^{\prime}\right)-\left\langle\bar{\lambda}_{2}+\lambda_{2}^{\prime}, v_{2}^{\prime}\right\rangle_{\Gamma}=L_{2}\left(v_{2}^{\prime}\right) \quad \forall v_{2}^{\prime}$,

$\left\langle\bar{\mu}_{1}, \bar{\gamma}+\gamma^{\prime}-\bar{u}_{1}-u_{1}^{\prime}\right\rangle_{\Gamma}=0 \quad \forall \bar{\mu}_{1}$,

$\left\langle\mu_{1}^{\prime}, \bar{\gamma}+\gamma^{\prime}-\bar{u}_{1}-u_{1}^{\prime}\right\rangle_{\Gamma}=0 \quad \forall \mu_{1}^{\prime}$,

$\left\langle\bar{\mu}_{2}, \bar{\gamma}+\gamma^{\prime}-\bar{u}_{2}-u_{2}^{\prime}\right\rangle_{\Gamma}=0 \quad \forall \bar{\mu}_{2}$,

$\left\langle\mu_{2}^{\prime}, \bar{\gamma}+\gamma^{\prime}-\bar{u}_{2}-u_{2}^{\prime}\right\rangle_{\Gamma}=0 \quad \forall \mu_{2}^{\prime}$,

$\left\langle\bar{\kappa}, \bar{\lambda}_{1}+\lambda_{1}^{\prime}+\bar{\lambda}_{2}+\lambda_{2}^{\prime}\right\rangle_{\Gamma}=0 \quad \forall \bar{\kappa}$,

$\left\langle\kappa^{\prime}, \bar{\lambda}_{1}+\lambda_{1}^{\prime}+\bar{\lambda}_{2}+\lambda_{2}^{\prime}\right\rangle_{\Gamma}=0 \quad \forall \kappa^{\prime}$.

In these equations, $\lambda_{i}=\bar{\lambda}_{i}+\lambda_{j}^{\prime} \in F$ are the fluxes computed from the side of $\Omega_{i}$ and $\mu_{i}=\bar{\mu}_{i}+\mu_{i}^{\prime} \in F$ the corresponding test functions $(i=1,2)$. The test function for the trace of the unknown $\gamma=\bar{\gamma}+\gamma^{\prime} \in T$ is denoted by $\kappa=\bar{\kappa}+\kappa^{\prime} \in T$. The boundary terms in (4)-(7) correspond to the weak imposition of fluxes on $\Gamma$, Eqs. (8) $-(11)$ to the weak continuity of $u_{i}=\bar{u}_{i}+u_{i}^{\prime}$ on $\Gamma(i=1,2)$ and

Eqs. (12) and (13) to the weak continuity of fluxes on $\Gamma$.

The previous formulation can be considered a straightforward extension of the classical three field formulation for $u, \gamma$ and $\lambda$, obtained by a splitting of the spaces where these unknowns belong (see [23) for a three field formulation of the equation). Our particular formulation the-fluxes of $\bar{u}$ to be $\bar{\lambda}_{i}=n_{i} \cdot\left(k \nabla \bar{u}_{i}+d \bar{u}_{i}\right)$ to $\Gamma$ from $\Omega_{i}$, and $\bar{\gamma}=\left.\bar{u}_{i}\right|_{\Gamma}(i=1,2)$. In ot fluxes and the continuity of $\bar{u}$ as in the tion (3), but treat $u^{\prime}, \gamma^{\prime}$ and $\lambda^{\prime}$ as in the st approach in particular implies

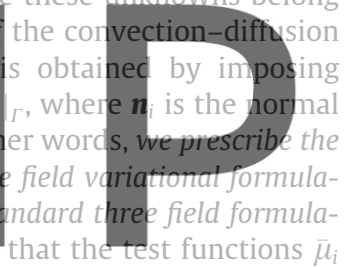
must be of the form $\bar{\mu}_{i}=\left.\boldsymbol{n}_{i} \cdot\left(k \nabla \bar{v}_{i}+\boldsymbol{a} \bar{v}_{i}\right)\right|_{\Gamma}$, for $\bar{v}_{i} \in \bar{V}_{i}$, and

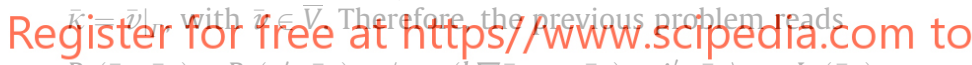

$B_{1}\left(\bar{u}_{1}, \bar{v}_{1}\right)+B_{1}\left(u_{1}^{\prime}, \bar{v}_{1}\right)-\left\langle\boldsymbol{n}_{1} \cdot\left(k \nabla \bar{u}_{1}+\boldsymbol{a} \bar{u}_{1}\right)+\lambda_{1}^{\prime}, \bar{v}_{1}\right\rangle_{\Gamma}=L_{1}\left(\bar{v}_{1}\right)$ $\forall \bar{v}_{1}$,

$B_{1}\left(\bar{u}_{1}, v_{1}^{\prime}\right)+B_{1}\left(u_{1}^{\prime}, v_{1}^{\prime}\right)-\left\langle\boldsymbol{n}_{1} \cdot\left(k \nabla \bar{u}_{1}+\boldsymbol{a} \bar{u}_{1}\right)+\lambda_{1}^{\prime}, v_{1}^{\prime}\right\rangle_{\Gamma}=L_{1}\left(v_{1}^{\prime}\right)$ $\forall v_{1}^{\prime}$,

$B_{2}\left(\bar{u}_{2}, \bar{v}_{2}\right)+B_{2}\left(u_{2}^{\prime}, \bar{v}_{2}\right)-\left\langle\boldsymbol{n}_{2} \cdot\left(k \nabla \bar{u}_{2}+\boldsymbol{a} \bar{u}_{2}\right)+\lambda_{2}^{\prime}, \bar{v}_{2}\right\rangle_{\Gamma}=L_{2}\left(\bar{v}_{2}\right)$ $\forall \bar{v}_{2}$,

$B_{2}\left(\bar{u}_{2}, v_{2}^{\prime}\right)+B_{2}\left(u_{2}^{\prime}, v_{2}^{\prime}\right)-\left\langle\boldsymbol{n}_{2} \cdot\left(k \nabla \bar{u}_{2}+\boldsymbol{a} \bar{u}_{2}\right)+\lambda_{2}^{\prime}, v_{2}^{\prime}\right\rangle_{\Gamma}=L_{2}\left(v_{2}^{\prime}\right)$ $\forall v_{2}^{\prime}$,

$\left\langle\boldsymbol{n}_{1} \cdot\left(k \nabla \bar{v}_{1}+\boldsymbol{a} \bar{v}_{1}\right), \gamma^{\prime}-u_{1}^{\prime}\right\rangle_{\Gamma}=0 \quad \forall \bar{v}_{1}$,

$\left\langle\mu_{1}^{\prime}, \gamma^{\prime}-u_{1}^{\prime}\right\rangle_{\Gamma}=0 \quad \forall \mu_{1}^{\prime}$,

$\left\langle\boldsymbol{n}_{2} \cdot\left(k \nabla \bar{v}_{2}+\boldsymbol{a} \bar{v}_{2}\right), \gamma^{\prime}-u_{2}^{\prime}\right\rangle_{\Gamma}=0 \quad \forall \bar{v}_{2}$,

$\left\langle\mu_{2}^{\prime}, \gamma^{\prime}-u_{2}^{\prime}\right\rangle_{\Gamma}=0 \quad \forall \mu_{2}^{\prime}$,

$\left\langle\bar{\kappa}, \boldsymbol{n}_{1} \cdot\left(k \nabla \bar{u}_{1}+\boldsymbol{a} \bar{u}_{1}\right)+\boldsymbol{n}_{2} \cdot\left(k \nabla \bar{u}_{2}+\boldsymbol{a} \bar{u}_{2}\right)+\lambda_{1}^{\prime}+\lambda_{2}^{\prime}\right\rangle_{\Gamma}=0 \quad \forall \bar{\kappa},(22)$

$\left\langle\kappa^{\prime}, \boldsymbol{n}_{1} \cdot\left(k \nabla \bar{u}_{1}+\boldsymbol{a} \bar{u}_{1}\right)+\boldsymbol{n}_{2} \cdot\left(k \nabla \bar{u}_{2}+\boldsymbol{a} \bar{u}_{2}\right)+\lambda_{1}^{\prime}+\lambda_{2}^{\prime}\right\rangle_{\Gamma}=0 \quad \forall \kappa^{\prime}$.

Adding up (14) and (16) and using (22) yields the original variational equation projected onto $\bar{V}$, that is to say

$B(\bar{u}, \bar{v})+B\left(u^{\prime}, \bar{v}\right)=L(\bar{v}) \quad \forall \bar{v}$.

It is understood that $B\left(u^{\prime}, \bar{v}\right)=B_{1}\left(u_{1}^{\prime}, \bar{v}_{1}\right)+B_{2}\left(u_{2}^{\prime}, \bar{v}_{2}\right)$. Integrating these terms by parts and using (18) and (20) we get

$B(\bar{u}, \bar{v})+\sum_{i=1}^{2}\left\langle u^{\prime}, \mathscr{L}^{*} \bar{v}\right\rangle_{\Omega_{i}}+\sum_{i=1}^{2}\left\langle\gamma^{\prime}, \boldsymbol{n}_{i} \cdot\left(k \nabla \bar{v}_{i}+\boldsymbol{a} \bar{v}_{i}\right)\right\rangle_{\Gamma}=L(\bar{v})$,

where

$\mathscr{L}^{*} \bar{v}:=-k \Delta \bar{v}-\boldsymbol{a} \cdot \nabla \bar{v}+s \bar{v}$ is the formal adjoint of $\mathscr{L}$. Adding up (15) and (17) and integrating the first terms by parts we get

$$
\begin{gathered}
\sum_{i=1}^{2}\left(B_{i}\left(\bar{u}, v^{\prime}\right)+B_{i}\left(u^{\prime}, v^{\prime}\right)-\left\langle\boldsymbol{n}_{i} \cdot\left(k \nabla \bar{u}_{i}+\boldsymbol{a} \bar{u}_{i}\right)+\lambda_{i}^{\prime}, v_{i}^{\prime}\right\rangle_{\Gamma}\right) \\
=\sum_{i=1}^{2}\left(\left\langle\mathscr{L} \bar{u}, v^{\prime}\right\rangle_{\Omega_{i}}+B_{i}\left(u^{\prime}, v^{\prime}\right)-\left\langle\lambda_{i}^{\prime}, v_{i}^{\prime}\right\rangle_{\Gamma}\right)=\sum_{i=1}^{2} L_{i}\left(v^{\prime}\right) .
\end{gathered}
$$

It is understood in this equation that $\left.(\cdot)\right|_{\Omega_{i}}=\left(\cdot \overline{)}_{i}\right.$. The final problem can be written as (24), (25), (23) and the addition of (19) and (21), that is to say

$$
\begin{aligned}
& B(\bar{u}, \bar{v})+\sum_{i=1}^{2}\left\langle u^{\prime}, \mathscr{L}^{*} \bar{v}\right\rangle_{\Omega_{i}}+\sum_{i=1}^{2}\left\langle\gamma^{\prime}, \boldsymbol{n}_{i} \cdot\left(k \nabla \bar{v}_{i}+\boldsymbol{a} \bar{v}_{i}\right)\right\rangle_{\Gamma}=L(\bar{v}) \forall \bar{v} \\
& \sum_{i=1}^{2}\left\langle\mathscr{L} \bar{u}, v^{\prime}\right\rangle_{\Omega_{i}}+B\left(u^{\prime}, v^{\prime}\right)-\sum_{i=1}^{2}\left\langle\lambda_{i}^{\prime}, v_{i}^{\prime}\right\rangle_{\Gamma}=L\left(v^{\prime}\right) \quad \forall v^{\prime}
\end{aligned}
$$

$$
\begin{aligned}
& \sum_{i=1}^{2}\left\langle\kappa^{\prime}, \boldsymbol{n}_{i} \cdot\left(k \nabla \bar{u}_{i}+\boldsymbol{a} \bar{u}_{i}\right)+\lambda_{i}^{\prime}\right\rangle_{\Gamma}=0 \quad \forall \boldsymbol{K}^{\prime}, \\
& \sum_{i=1}^{2}\left\langle\mu_{i}^{\prime}, \gamma^{\prime}-u_{i}^{\prime}\right\rangle_{\Gamma}=0 \quad \forall \mu_{1}^{\prime}, \mu_{2}^{\prime} .
\end{aligned}
$$

This is the four field formulation we were looking for. Its importance relies on the fact that it is the theoretical framework to develop approximations in which $u$ is split into a contribution which is continuous on $I$ and another one which is discontinuous. Obviously, this formulation is

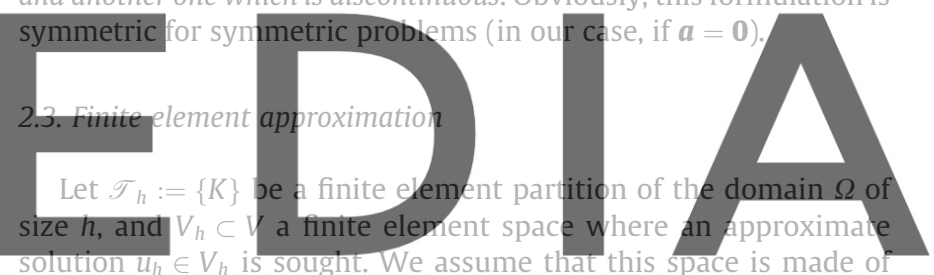

continuous functions. To simplify the analysis, we will assume that

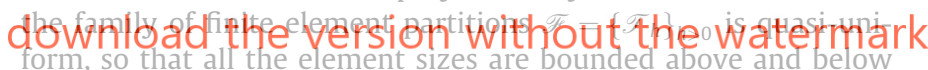

by constants multiplied by $h$. We will also use the abbreviations

$\|\cdot\| L^{2}(K) \equiv\|\cdot\|_{K}$ and $\|\cdot\|_{L^{2}(\partial K)} \equiv\|\cdot\|_{\partial K} \cdot$

Consider the previous setting with $\bar{V}=V_{h}$, and therefore $V=V_{h} \oplus V^{\prime}$, with $V^{\prime}$ to be defined, and $u=u_{h}+u^{\prime}, v=v_{h}+v^{\prime}$. In order to focus our attention to the expression for the subscales on the interelement boundaries, we will not specify the choice for $V^{\prime}$, which depends on the particular VMS approximation used.

As before, let also $\gamma^{\prime}$ be the trace of $u^{\prime}$ on the interelement boundaries and $\lambda^{\prime}$ the flux, being the corresponding spaces $T^{\prime}$ and $F^{\prime}$, and the corresponding test functions $\kappa^{\prime} \in T^{\prime}$ and $\mu^{\prime} \in F^{\prime}$. According to the four field formulation presented in the previous subsection, now considering $\Omega$ split into the element domains of the finite element partition, the variational problem (3) is exactly equivalent to find $u_{h} \in V_{h}, u^{\prime} \in V^{\prime}, \gamma^{\prime} \in T^{\prime}$ and $\lambda^{\prime} \in F^{\prime}$ such that

$$
\begin{aligned}
& B\left(u_{h}, v_{h}\right)+\sum_{K}\left\langle u^{\prime}, \mathscr{L}^{*} v_{h}\right\rangle_{K}+\sum_{K}\left\langle\gamma^{\prime}, k \partial_{n} v_{h}\right\rangle_{\partial K}=L\left(v_{h}\right) \forall v_{h} \in V_{h}, \\
& \sum_{K}\left\langle\mathscr{L} u_{h}, v^{\prime}\right\rangle_{K}+B\left(u^{\prime}, v^{\prime}\right)-\sum_{K}\left\langle\lambda^{\prime}, v^{\prime}\right\rangle_{\partial K}=L\left(v^{\prime}\right) \quad \forall v^{\prime} \in V^{\prime}, \\
& \sum_{K}\left\langle\kappa^{\prime}, k \partial_{n} u_{h}+\lambda^{\prime}\right\rangle_{\partial K}=0 \quad \forall \kappa^{\prime} \in T^{\prime}, \\
& \sum_{K}\left\langle\mu^{\prime}, \gamma^{\prime}-u^{\prime}\right\rangle_{\partial K}=0 \quad \forall \mu^{\prime} \in F^{\prime} .
\end{aligned}
$$

Note that the jumps of the convective fluxes are zero because of the continuity assumed for the finite element functions.

The approximation process consists of different ingredients, all aiming at giving a closed problem for $u_{h}$ alone. For that we will propose heuristic approximations for $\gamma^{\prime}$ and $\lambda^{\prime}$ and then we will perform a stability analysis to check that the resulting formulation 
is stable. Let us insist that, up to this point, problem (30)-(33) is exact. Furthermore, for $u_{h}=0$ it could be used as the variational framework to develop discontinuous Galerkin approximations (see Remark 2 below).

\subsection{Subscales on the element boundaries}

Let us consider for simplicity the 2D case and the situation depicted in Fig. 1, where two elements $K_{1}$ and $K_{2}$ share an edge $E$ (recall that $E$ stands for "edge" in 2D or face in 3D). Unless otherwise indicated (see Remark 1 below), all the edges are considered interior, that is to say, the element boundaries on $\partial \Omega$ are excluded.

Let $u_{i}^{\prime}$ be the subscale approximated in the interior of element $K_{i}, i=1,2$. We assume that this approximation is valid up to a distance $\delta$ to the element boundary. This distance will be taken of the form $\delta=\delta_{0} h$, with $0 \leqslant \delta_{0} \leqslant 1 / 2$.

Approximation of $\lambda^{\prime}$.

The values of $\lambda^{\prime}$ on $\partial K$ are weak approximations to the fluxes of $u^{\prime}$. Given the trace $\gamma^{\prime}$ of this unknown, we delete (33) and propose the following closed form expression for $\lambda^{\prime}$ :

\section{$\lambda_{\partial K_{i} \cap E}^{\prime} \approx k \frac{\gamma_{E}^{\prime}-u_{i}^{\prime}}{\delta}, \quad i=1,2$}

where now $u_{i}^{\prime}$ has to be understood as the subscale computed in the element interiors and evaluated at edge $E$. We want to remark that, apart from the assumptions inherent to the VMS framework and the imposition of the transmission conditions (see below), this is the only approximation we really require to compute the subscales on the inferelement boundaries. Obviously, other finite-difference-like approximations to the fluxes of the Approximation of $\gamma^{\prime}$

Eq (32) states the weak continuity of the total fluxes on the element boundaries. The idea now is prescription of this $\left.n_{2} g\right|_{a K_{2}}$ denotes the juinn replace this equation by an f a scalar function $g$ across edge $E$ and $\llbracket \partial_{n} g \rrbracket_{E}=\left.\boldsymbol{n}_{1} \cdot \nabla g\right|_{\partial K_{1} \cap E}+\left.\boldsymbol{n}_{2} \cdot \nabla g\right|_{\partial K_{2} \cap E}$ the jump of the Registeflifori free at https//WWW.scipedia.com to

$$
\begin{aligned}
0 & =\llbracket k \partial_{n} u \rrbracket_{E}=\llbracket k \partial_{n} u_{h} \rrbracket_{E}+\lambda_{\partial K_{1} \cap E}^{\prime}+\lambda_{\partial K_{2} \cap E}^{\prime} \\
& \approx \llbracket k \partial_{n} u_{h} \rrbracket_{E}+k \frac{\gamma_{E}^{\prime}-u_{1}^{\prime}}{\delta}+k \frac{\gamma_{E}^{\prime}-u_{2}^{\prime}}{\delta} .
\end{aligned}
$$

From this expression, and for $k$ constant, we obtain the approximation we were looking for

$$
\gamma_{E}^{\prime} \approx\left\{u^{\prime}\right\}_{E}-\frac{\delta}{2} \llbracket \partial_{n} u_{h} \rrbracket_{E}
$$

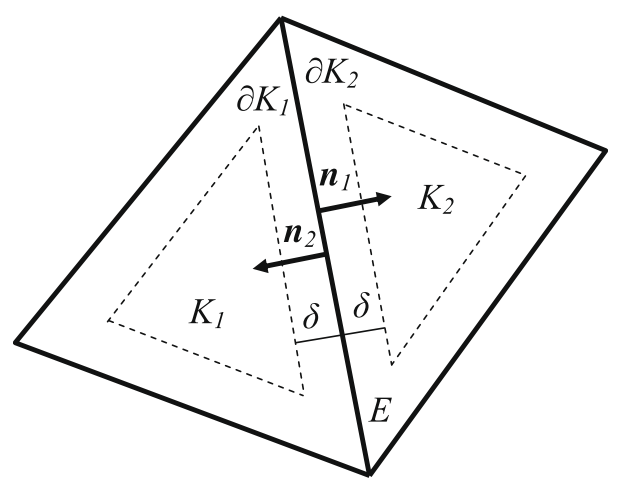

Fig. 1. Notation for the approximation of the subscales on the element boundaries. where $\left\{u^{\prime}\right\}_{E}:=\frac{1}{2}\left(u_{1}^{\prime}+u_{2}^{\prime}\right)$ is the average of the subscales computed in the element interiors evaluated at edge $E$. From (36) it is observed that $\delta_{0}$ will play the role of an algorithmic parameter for which, following our approach, we have a geometrical interpretation.

From now onwards we will use the symbol $=$ instead of $\approx$, understanding that in some places we perform approximation (34) that has led us to (36).

Remark 1. (Neumann boundary conditions). Suppose that $F_{K}=\partial K \cap \partial \Omega$ and that instead of the Dirichlet condition (2) the Neumann condition $-k \partial_{n} u=q$ is prescribed. In this case, (35) should be replaced by

$q=-\left.k \partial_{n} u_{h}\right|_{F_{K}}-k \frac{\gamma_{F_{K}}^{\prime}-u_{K}^{\prime}}{\delta}$,

so that the contribution to $L$ in (3) that would appear due to the Neumann condition would be modified by the approximation to the subscale on the boundary, and there would be also a contribution to the bilinear form $B$. We will come back to this point in the case of the Stokes problem, where this fact has more important consequences.

\section{Problem for $u_{h}$ and $u^{\prime}$}

From (36) we obtain the following approximation for the fluxes of the subscales:

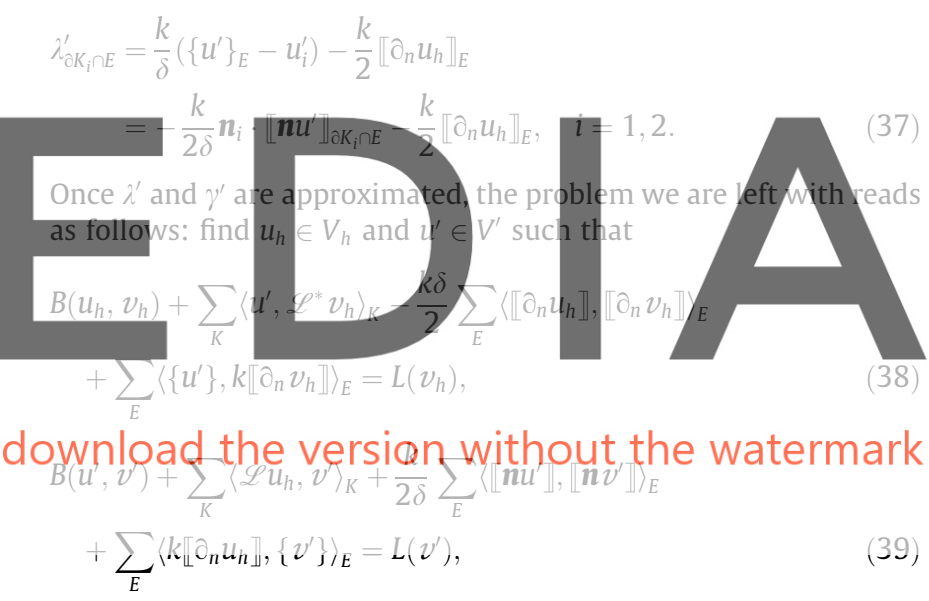

for all $v_{h} \in V_{h}$ and $v^{\prime} \in V^{\prime}$.

Remark 2. Observe that this system of variational equations can be understood as a general framework to approximate unknowns with a continuous part $\left(u_{h}\right)$ and an approximated discontinuous part $\left(u^{\prime}\right)$. Furthermore, if the continuous part is zero, we are left with (39) with $u_{h}=0$, which corresponds to the classical Galerkin method enforcing continuity across interelement boundaries through Nitsche's method, although with approximation (37) for the fluxes, so that the classical terms involving $\partial_{n} u^{\prime}$ and $\partial_{n} v^{\prime}$ are missing (see $[2,15]$ ). For piecewise constant approximations these terms would not appear, and we would obtain a classical piecewise-constant discontinuous Galerkin approximation.

\subsection{Subscales in the element interiors}

Up to now we have replaced variational equations for the fluxes of the subscales and their traces by approximated closed form expressions. It can be seen from problem (38) and (39) that the resulting formulation is symmetric for symmetric problems. However, now we will use an additional approximation that will make the problem loose its symmetry, but that will greatly simplify the implementation of the formulation. This approximation is inherent to all VMS formulations to yield a closed form expression for the subscales in the element interiors. 
If we integrate the second term in the left-hand-side (LHS) of (31) by parts we get

$B\left(u^{\prime}, v^{\prime}\right)=\sum_{K}\left\langle\mathscr{L} u^{\prime}, v^{\prime}\right\rangle_{K}+\sum_{K}\left\langle k \partial_{n} u^{\prime}, v^{\prime}\right\rangle_{\partial K}$

If instead of using (37) we assume that $\lambda^{\prime}$ approximates $k \partial_{n} u^{\prime}$, the second term in this last expression cancels with the third one in the LHS of (31). Therefore, the final problem is: find $u_{h} \in V_{h}$ and $u^{\prime} \in V^{\prime}$ such that

$B\left(u_{h}, v_{h}\right)+\sum_{K}\left\langle u^{\prime}, \mathscr{L}^{*} v_{h}\right\rangle_{K}+\sum_{E}\left\langle\left\{u^{\prime}\right\}-\frac{\delta}{2} \llbracket \partial_{n} u_{h} \rrbracket, k \llbracket \partial_{n} v_{h} \rrbracket\right\rangle_{E}=L\left(v_{h}\right)$,

$\sum_{K}\left\langle\mathscr{L} u_{h}, v^{\prime}\right\rangle_{K}+\sum_{K}\left\langle\mathscr{L} u^{\prime}, v^{\prime}\right\rangle_{K}=L\left(v^{\prime}\right)$

for all $v_{h} \in V_{h}$ and $v^{\prime} \in V^{\prime}$. The last term in the LHS of (40) is the main novelty with respect to classical stabilized finite element methods designed in the variational multiscale framework.

Remark 3. Note that if in (39) $v^{\prime}$ is considered continuous we obtain (41) with no additional approximation. In other words, if the subscale is approximated with a Petrov-Galerkin method (leading to a non-symmetric formulation) in which the space of test functions is continuous, we recover (41). This is not however the approach we will adopt.

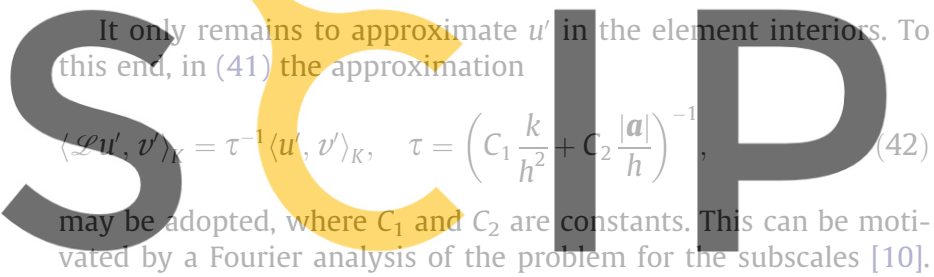

In particular, it implies that the subscales in the element interiors

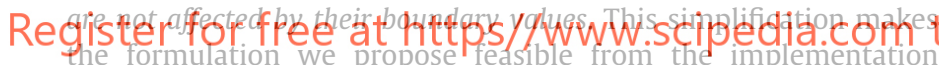
standpoint. Let us stress once again that this approximation is not original of this work, but common to all VMS methods that compute locally the subscales in the element interiors.

Once all the approximations are made, the final problem is to find $u_{h} \in V_{h}$ and $u^{\prime} \in V^{\prime}$ such that

$B\left(u_{h}, v_{h}\right)+\sum_{K}\left\langle u^{\prime}, \mathscr{L}^{*} v_{h}\right\rangle_{K}+\sum_{E}\left\langle\left\{u^{\prime}\right\}-\frac{\delta}{2} \llbracket \partial_{n} u_{h} \rrbracket, k \llbracket \partial_{n} v_{h} \rrbracket\right\rangle_{E}=L\left(v_{h}\right)$,

$\sum_{K}\left\langle\mathscr{L} u_{h}, v^{\prime}\right\rangle_{K}+\sum_{K} \tau^{-1}\left\langle u^{\prime}, v^{\prime}\right\rangle_{K}=L\left(v^{\prime}\right)$

for all $v_{h} \in V_{h}$ and $v^{\prime} \in V^{\prime}$.

The variational Eq. (44) automatically yields and expression for the subscales in the element interiors in terms of the finite element component, provided $V^{\prime}$ is approximated by a space of discontinuous functions. For constant $\tau$ it implies that

$u^{\prime}=\tau P_{V^{\prime}}\left(f-\mathscr{L} u_{h}\right)$

where $P_{V^{\prime}}$ is the projection onto $V^{\prime}$. However, it will be convenient for the following analysis to keep $u^{\prime}$ as unknown of the problem. Particular cases of projection that fit into the present framework are the orthogonal subscales stabilization (OSS) proposed in [9] and the algebraic version of the subgrid-scale stabilization (ASGS) (see $[8,16]$ ), where $P_{V^{\prime}}$ is the identity (at least when applied to $\left.f-\mathscr{L} u_{h}\right)$. The expression of $\tau(42)$ is in fact not important, except for a condition on constant $C_{1}$ indicated later.

\subsection{Stability analysis}

Let us consider the bilinear form of the problem in $\left(V_{h} \times V^{\prime}\right) \times\left(V_{h} \times V^{\prime}\right)$ :

$$
\begin{gathered}
B_{\exp }\left(u_{h}, u^{\prime} ; v_{h}, v^{\prime}\right):=B\left(u_{h}, v_{h}\right)+\sum_{K}\left\langle u^{\prime}, \mathscr{L}^{*} v_{h}\right\rangle_{K}+\sum_{E}\left\langle\left\{u^{\prime}\right\}, k \llbracket \partial_{n} v_{h} \rrbracket\right\rangle_{E} \\
-\frac{\delta}{2} \sum_{E}\left\langle\llbracket \partial_{n} u_{h} \rrbracket, k \llbracket \partial_{n} v_{h} \rrbracket\right\rangle_{E}+\sum_{K}\left\langle\mathscr{L} u_{h}, v^{\prime}\right\rangle_{K}+\sum_{K} \tau^{-1}\left\langle u^{\prime}, v^{\prime}\right\rangle_{K} .
\end{gathered}
$$

Let us prove stability of the problem by showing that $B_{\exp }$ is coercive in a certain norm. We have that

$$
\begin{aligned}
B_{\exp }\left(u_{h}, u^{\prime} ; u_{h}, u^{\prime}\right) & \\
= & B\left(u_{h}, u_{h}\right)+\sum_{K}\left\langle u^{\prime}, \mathscr{L}^{*} u_{h}+\mathscr{L} u_{h}\right\rangle_{K}+\sum_{E}\left\langle\left\{u^{\prime}\right\}, k \llbracket \partial_{n} u_{h} \rrbracket\right\rangle_{E} \\
& -\frac{\delta}{2} \sum_{E} k\left\|\llbracket \partial_{n} u_{h} \rrbracket\right\|_{E}^{2}+\sum_{K} \tau^{-1}\left\|u^{\prime}\right\|_{K}^{2} \geqslant k\left\|\nabla u_{h}\right\|^{2}+s\left\|u_{h}\right\|^{2} \\
& -\sum_{K}\left\|u^{\prime}\right\|_{K}\left\|-2 k \Delta u_{h}+2 s u_{h}\right\|_{K}-\sum_{E}\left\|\left\{u^{\prime}\right\}\right\|_{E} k\left\|\llbracket \partial_{n} u_{h} \rrbracket\right\|_{E}
\end{aligned}
$$

We assume now that the classical inverse estimates

$$
\left\|\Delta v_{h}\right\|_{K}^{2} \leqslant \frac{C_{\text {inv }}}{h^{2}}\left\|\nabla v_{h}\right\|_{K}^{2}, \quad\left\|v_{h}\right\|_{L^{\infty}(K)}^{2} \leqslant \frac{C_{\text {inv }}}{h^{d}}\left\|v_{h}\right\|_{K}^{2} \quad \forall v_{h} \in V_{h},
$$

hold true (see $[13,5]$ ). In particular, the second, which also holds for derivatives of finite element functions, implies the trace inequality
$\left\|v_{h}\right\|_{\partial K}^{2} \leqslant C_{\mathrm{tr}} h^{-1}\left\|v_{h}\right\|_{K}^{2}$
which applied to $\partial_{n} v_{h}$ yields
$\left\|\hat{D}_{h} v_{h}\right\|_{\text {aK }}^{2} \leqslant C_{\mathrm{tr}} h^{-1}\left\|\nabla v_{h}\right\|_{K}^{2}$.
Using these inverse estimates, we have (see Pig. 1 for he notation).

\section{dơwnkoâd the versiblon whithoûth the watermark}

$$
\begin{aligned}
& \geqslant-\frac{\delta}{2} \sum_{K} 2 k\left\|\partial_{n} u_{h}\right\|_{\partial K}^{2} \\
& \geqslant-\frac{\delta_{0} h}{2} \sum_{K} 2 k C_{\mathrm{tr}} h^{-1}\left\|\nabla u_{h}\right\|_{K}^{2}=-\delta_{0} C_{\mathrm{tr}} k\left\|\nabla u_{h}\right\|^{2} .
\end{aligned}
$$

Let us obtain a working inequality. Let $a$ and $b$ be discontinuous positive functions defined on the finite element partition. Using the notation $a_{i}:=\left.a\right|_{\partial K_{i} \cap E}$, for any $\beta>0$ we have that

$$
\begin{aligned}
\sum_{E}\left(a_{1}+a_{2}\right)\left(b_{1}+b_{2}\right) & \leqslant \sum_{E} \frac{h}{2 \beta}\left(a_{1}+a_{2}\right)^{2}+\sum_{E} \frac{\beta}{2 h}\left(b_{1}+b_{2}\right)^{2} \\
& \leqslant \sum_{E} \frac{h}{\beta}\left(a_{1}^{2}+a_{2}^{2}\right)+\sum_{E} \frac{\beta}{h}\left(b_{1}^{2}+b_{2}^{2}\right) \\
& \leqslant\left.\sum_{K} \frac{h}{\beta} a\right|_{\partial K} ^{2}+\left.\sum_{K} \frac{\beta}{h} b\right|_{\text {วK }} ^{2} .
\end{aligned}
$$

Now we make the assumption that the subscales are such that the inverse estimates also hold for them. Using the previous inequality we obtain, for any $\beta_{3}>0$ :

$$
\begin{aligned}
& -\sum_{E}\left\|\left\{u^{\prime}\right\}\right\|_{E} k\left\|\llbracket \partial_{n} u_{h} \rrbracket\right\|_{E} \\
& =-\sum_{E} k \frac{1}{2}\left\|u_{1}^{\prime}+u_{2}^{\prime}\right\|_{E}\left\|\left.\partial_{n} u_{h}\right|_{\partial K_{1} \cap E}+\left.\partial_{n} u_{h}\right|_{\partial K_{2} \cap E}\right\|_{E} \\
& \geqslant-\sum_{K} \frac{\beta_{3}}{2} C_{\text {tr }} \frac{k}{h^{2}}\left\|u^{\prime}\right\|_{K}^{2}-\sum_{K} \frac{1}{2 \beta_{3}} C_{\text {tr }} k\left\|\nabla u_{h}\right\|_{K}^{2} .
\end{aligned}
$$


Using the bounds obtained, it follows that

$$
\begin{aligned}
& B_{\exp }\left(u_{h}, u^{\prime} ; u_{h}, u^{\prime}\right) \\
& \geqslant k\left\|\nabla u_{h}\right\|^{2}+s\left\|u_{h}\right\|^{2}-\sum_{K}\left\|u^{\prime}\right\|_{K} 2 k \frac{C_{\text {inv }}^{1 / 2}}{h}\left\|\nabla u_{h}\right\|_{K} \\
&-\sum_{K}\left\|u^{\prime}\right\|_{K} 2 s\left\|u_{h}\right\|_{K}-\delta_{0} C_{\mathrm{tr}} k\left\|\nabla u_{h}\right\|^{2}-\sum_{E}\left\|\left\{u^{\prime}\right\}\right\|_{E} k\left\|\llbracket \partial_{n} u_{h} \rrbracket\right\|_{E} \\
&+\sum_{K} \tau^{-1}\left\|u^{\prime}\right\|_{K}^{2} \geqslant k\left\|\nabla u_{h}\right\|^{2}+s\left\|u_{h}\right\|^{2} \\
&-\sum_{K}\left(\beta_{1} k \frac{C_{\text {inv }}}{h^{2}}\left\|u^{\prime}\right\|_{K}^{2}+\frac{1}{\beta_{1}} k\left\|\nabla u_{h}\right\|_{K}^{2}\right) \\
&-\sum_{K}\left(\beta_{2} s\left\|u^{\prime}\right\|_{K}^{2}+\frac{1}{\beta_{2}} s\left\|u_{h}\right\|_{K}^{2}\right) \\
&-\sum_{K} \frac{\beta_{3}}{2} C_{\mathrm{tr}} \frac{k}{h^{2}}\left\|u^{\prime}\right\|_{K}^{2}-\sum_{K} \frac{1}{2 \beta_{3}} C_{\mathrm{tr}} k\left\|\nabla u_{h}\right\|_{K}^{2}
\end{aligned}
$$$$
-\delta_{0} C_{\mathrm{tr}} k\left\|\nabla u_{h}\right\|^{2}+\sum \tau^{-1}\left\|u^{\prime}\right\|_{K}^{2}
$$

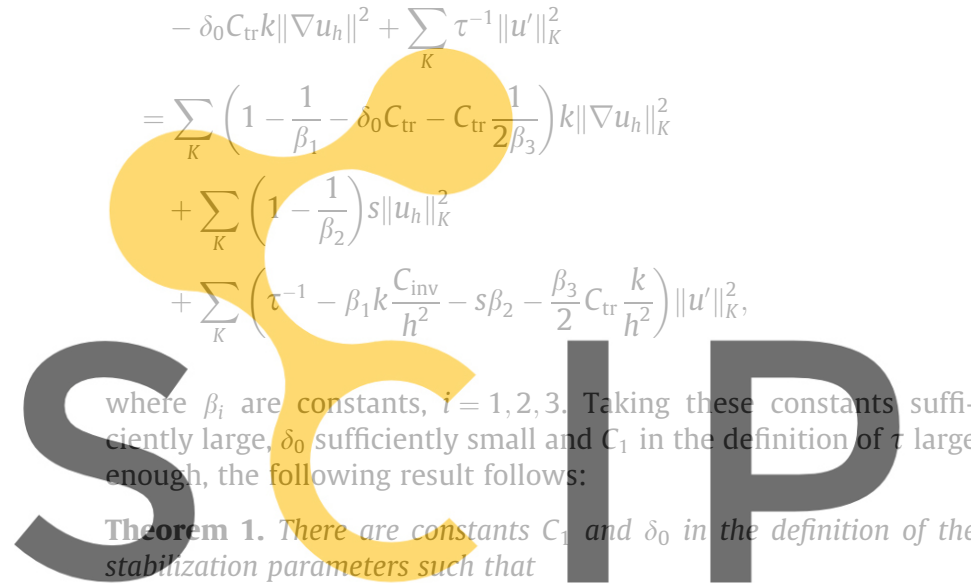

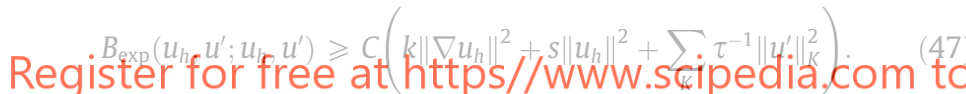

Remark 4. Let us enumerate the essential ideas and highlight the original aspects of the analysis presented in this section:

- The driving idea is that the subscales on the boundary are determined by the transmission condition. In the case of the CDR equation and using continuous interpolations, this is the continuity of the diffusive fluxes.

- The essential approximation to make the problem computationally viable is to compute the subscales in the element interiors without taking into account their values on the boundaries.

- In the stability analysis presented, the subscales have their own "personality". They appear explicitly in the stability estimate. The final stability estimate for the finite element unknown depends on the way the subscales are approximated (that is to say, on how $V^{\prime}$ is chosen).

- It is observed that the expression of $\tau$ in terms of $\boldsymbol{a}$ is not used in the stability analysis. However, it is required in the convergence analysis.

- The only thing we have shown is that the terms introduced by the boundary contribution from the subscales can be controlled, but there seems to be no gain in considering the subscales on the boundaries. The stability estimate (47) is the same that would be obtained without the last term in the LHS of (40) which is, as it has been said, the main novelty of our proposal.

As stated in the last item, subscales on the boundary do not improve stability for the CDR equation. This is not so for the Stokes problem analyzed next.

\section{Stokes problem}

\subsection{Problem statement and finite element approximation}

In this section, we turn our attention to the Stokes problem, which consists of finding a velocity $\boldsymbol{u}: \Omega \rightarrow \mathbb{R}^{d}$ and a pressure $p: \Omega \rightarrow \mathbb{R}$ such that

$$
\begin{aligned}
& -v \Delta \boldsymbol{u}+\nabla p=\boldsymbol{f} \text { in } \Omega \subset \mathbb{R}^{d}, \\
& \nabla \cdot \boldsymbol{u}=0 \text { in } \Omega, \\
& \boldsymbol{u}=\mathbf{0} \text { on } \partial \Omega .
\end{aligned}
$$

The purpose is to extend the ideas of the previous section to this problem. Let now $V=H_{0}^{1}(\Omega)^{d}, Q=L^{2}(\Omega) / \mathbb{R}$. The variational problem consists of finding $[\boldsymbol{u}, p] \in V \times Q$ such that

$$
\begin{aligned}
B([\boldsymbol{u}, p],[\boldsymbol{v}, q]) & :=v(\nabla \boldsymbol{u}, \nabla \boldsymbol{v})-(p, \nabla \cdot \boldsymbol{v})+(q, \nabla \cdot \boldsymbol{u}) \\
& =\langle\boldsymbol{f}, \boldsymbol{v}\rangle \quad \forall[\boldsymbol{v}, q] \in V \times Q .
\end{aligned}
$$

For the sake of simplicity, we will consider subscales only for the velocity, not for the pressure. Pressure subscales can be easily introduced (see [10]), but they do not contribute to the present discussion. It is also possible to derive a general framework as in the previous section, using the trace of the velocity subscales and their fluxes as additional variables, leading to a five field formulation, the five fields being velocity, velocity subscale, trace of velocity subscale, flux of velocity subscale and pressure. However, we may directly work with velocity, velocity subscale and pressure, understanding that the

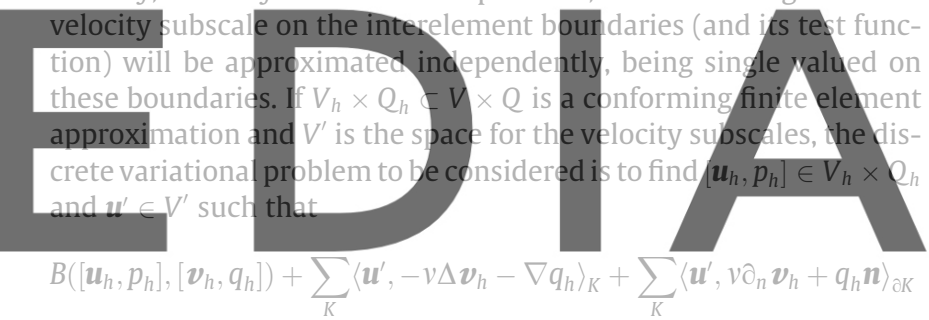

$$
\begin{aligned}
\sum_{K}\langle & \left.v\left(\partial_{n} \boldsymbol{u}_{h}+\partial_{n} \boldsymbol{u}^{\prime}\right)-p_{h} \boldsymbol{n}, \boldsymbol{v}^{\prime}\right\rangle_{\partial K}+\sum_{K}\left\langle-v \Delta \boldsymbol{u}_{h}+\nabla p_{h}, \boldsymbol{v}^{\prime}\right\rangle_{K} \\
& +\sum_{K}\left\langle-v \Delta \boldsymbol{u}^{\prime}, \boldsymbol{v}^{\prime}\right\rangle_{K}=\left\langle\boldsymbol{f}, \boldsymbol{v}^{\prime}\right\rangle,
\end{aligned}
$$

which must hold for all $\left[\boldsymbol{v}_{h}, q_{h}\right] \in V_{h} \times Q_{h}$ and all $\boldsymbol{v}^{\prime} \in V^{\prime}$. The first term in the second discrete variational equation must be zero because of the (weak) continuity of the stress normal to the element boundaries (recall that $\boldsymbol{v}^{\prime}$ has to be considered single valued when evaluated at the interelement boundaries).

As for the CDR equation, the approximation

$\left\langle-v \Delta \boldsymbol{u}^{\prime}, \boldsymbol{v}^{\prime}\right\rangle_{K}=\tau^{-1}\left\langle\boldsymbol{u}^{\prime}, \boldsymbol{v}^{\prime}\right\rangle_{K}, \quad \tau^{-1}=C_{1} \frac{v}{h^{2}}$

is adopted. Likewise, the subscale on the boundary will be approximated by an expression $\boldsymbol{u}_{E}^{\prime}$ to be determined, so that the problem to be solved is to find $\left[\boldsymbol{u}_{h}, p_{h}\right] \in V_{h} \times Q_{h}$ and $\boldsymbol{u}^{\prime} \in V^{\prime}$ such that

$$
\begin{aligned}
& B\left(\left[\boldsymbol{u}_{h}, p_{h}\right],\left[\boldsymbol{v}_{h}, q_{h}\right]\right)+\sum_{K}\left\langle\boldsymbol{u}^{\prime},-v \Delta \boldsymbol{v}_{h}-\nabla q_{h}\right\rangle_{K}+\sum_{K}\left\langle\boldsymbol{u}_{E}^{\prime}, v \partial_{h} \boldsymbol{v}_{h}+q_{h} \boldsymbol{n}\right\rangle_{\partial K} \\
& \quad=\left\langle\boldsymbol{f}, \boldsymbol{v}_{h}\right\rangle,
\end{aligned}
$$

$\sum_{K}\left\langle-v \Delta \boldsymbol{u}_{h}+\nabla p_{h}, \boldsymbol{v}^{\prime}\right\rangle_{K}+\sum_{K} \tau^{-1}\left\langle\boldsymbol{u}^{\prime}, \boldsymbol{v}^{\prime}\right\rangle_{K}=\left\langle\boldsymbol{f}, \boldsymbol{v}^{\prime}\right\rangle$,

which must hold for all $\left[\boldsymbol{v}_{h}, q_{h}\right] \in V_{h} \times Q_{h}$ and all $\boldsymbol{v}^{\prime} \in V^{\prime}$. The expression of $\tau$ is given in (48), but $\boldsymbol{u}_{E}^{\prime}$ is required to close the problem. 


\subsection{Subscales on the element boundaries}

The condition to determine the expression of the subscale velocity on the boundary is that the normal component of the stress be continuous across interelement boundaries. Using the same notation as in the previous section, this can be written as follows:

$$
\begin{aligned}
\mathbf{0} & =\llbracket-p \boldsymbol{n}+v \partial_{n} \boldsymbol{u} \rrbracket_{E}=\llbracket-p_{h} \boldsymbol{n}+v \partial_{n} \boldsymbol{u}_{h} \rrbracket_{E}+\llbracket v \partial_{n} \boldsymbol{u}^{\prime} \rrbracket_{E} \\
& =\llbracket-p_{h} \boldsymbol{n}+v \partial_{n} \boldsymbol{u}_{h} \rrbracket_{E}+\frac{v}{\delta}\left(2 \boldsymbol{u}_{E}^{\prime}-\boldsymbol{u}_{1}^{\prime}-\boldsymbol{u}_{2}^{\prime}\right),
\end{aligned}
$$

from where the approximation we propose is

$$
\boldsymbol{u}_{E}^{\prime}=\left\{\boldsymbol{u}^{\prime}\right\}_{E}-\frac{\delta}{2 v} \llbracket v \partial_{n} \boldsymbol{u}_{h}-p_{h} \boldsymbol{n} \rrbracket_{E}
$$

\section{which is the counterpart of (36) for the Stokes problem. Inserting} (51) into the discrete variational problem (49) and (50) results in

\section{$B\left(\left[\boldsymbol{u}_{h}, p_{h}\right],\left[\boldsymbol{v}_{h}, q_{h}\right]\right)+\sum_{K}\left\langle\boldsymbol{u}^{\prime},-v \Delta \boldsymbol{v}_{h}-\nabla q_{h}\right\rangle_{K}$ $\sum\left\langle\left\{\boldsymbol{u}^{\prime}\right\},\left[v \partial_{n} \boldsymbol{v}_{h}+q_{h} \boldsymbol{n} \rrbracket\right\rangle_{E}\right.$}

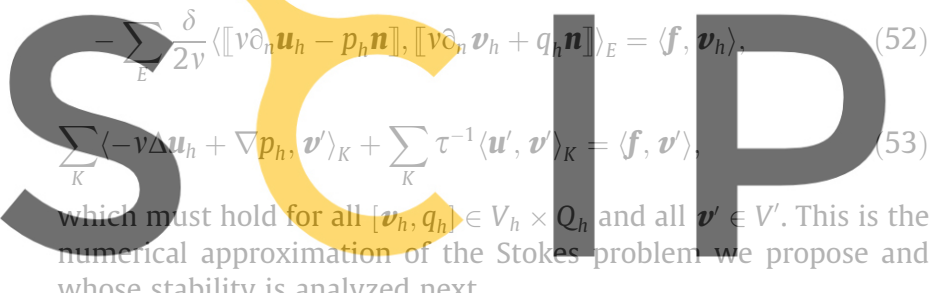

whose stability is analyzed next.

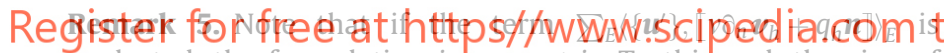
neglected, the formulation is symmetric. To this end, the sign of $q_{h}$ in the term $\left.\left\langle\llbracket v \partial_{n} \boldsymbol{u}_{h}-p_{h} \boldsymbol{n} \rrbracket\right], \llbracket v \partial_{n} \boldsymbol{v}_{h}+q_{h} \boldsymbol{n} \rrbracket\right\rangle_{\mathrm{E}}$ is essential. On the other hand, it seems reasonable to neglect $\left\{\boldsymbol{u}^{\prime}\right\}$ if discontinuous pressures are used because then the source of instability is known to be related to the lack of control on the pressure jumps. In particular, for the $P_{1} / P_{0}$ element used in the examples of Section 5, $\boldsymbol{u}^{\prime}=\mathbf{0}$ in the element interiors.

Remark 6. (Neumann boundary conditions). Suppose again that $F_{K}=\partial K \cap \partial \Omega$ and that the Neumann condition $-p \boldsymbol{n}+v \partial_{\boldsymbol{n}} \boldsymbol{u}=\boldsymbol{t}$ is prescribed. The subscale $\boldsymbol{u}_{F_{K}}^{\prime}$ should be computed from

$\boldsymbol{t}=-p_{h} \boldsymbol{n}+v \partial_{n} \boldsymbol{u}_{h}+\frac{v}{\delta}\left(\boldsymbol{u}_{F_{K}}^{\prime}-\boldsymbol{u}_{K}^{\prime}\right)$.

In this case, the terms

$\sum_{K}\left(\left\langle\boldsymbol{u}_{K}^{\prime}, v \partial_{n} \boldsymbol{v}_{h}+q_{h} \boldsymbol{n}\right\rangle_{F_{K}}-\frac{\delta}{v}\left\langle v \partial_{n} \boldsymbol{u}_{h}-p_{h} \boldsymbol{n}, v \partial_{n} \boldsymbol{v}_{h}+q_{h} \boldsymbol{n}\right\rangle_{F_{K}}\right)$,

and

$-\frac{\delta}{v} \sum_{K}\left\langle\boldsymbol{t}, v \partial_{n} \boldsymbol{v}_{h}+q_{h} \boldsymbol{n}\right\rangle_{F_{K}}$

should be added to the LHS and right-hand-side (RHS) of (52), respectively. Stability on these boundaries will be enhanced by the term $\sum_{K} \frac{\delta}{v}\left\langle p_{h}, q_{h}\right\rangle_{F_{K}}$. This approach might be important as well in fluid-structure interaction problems, where one of the problems (the structure for example) is computed using the normal stresses $t$ computed in the other domain. It is known that that in some situa- tions staggered coupled algorithms may suffer from the so called artificial mass effect due to the lack of stability in the imposition of the Neumann condition.

\subsection{Stability analysis}

As for the CDR equation, it is convenient to define the expanded bilinear form of problem (52) and (53), including the subscales as unknowns, which is

$$
\begin{aligned}
& B_{\exp }\left(\left[\boldsymbol{u}_{h}, p_{h}\right], \boldsymbol{u}^{\prime} ;\left[\boldsymbol{v}_{h}, q_{h}\right], \boldsymbol{v}^{\prime}\right) \\
& =B\left(\left[\boldsymbol{u}_{h}, p_{h}\right],\left[\boldsymbol{v}_{h}, q_{h}\right]\right)+\sum_{K}\left\langle\boldsymbol{u}^{\prime},-v \Delta \boldsymbol{v}_{h}-\nabla q_{h}\right\rangle_{K} \\
& +\sum_{K}\left\langle\boldsymbol{v}^{\prime},-v \Delta \boldsymbol{u}_{h}+\nabla p_{h}\right\rangle_{K}+\sum_{E}\left\langle\left\{\boldsymbol{u}^{\prime}\right\}, \llbracket v \partial_{n} \boldsymbol{v}_{h}+q_{h} \boldsymbol{n} \rrbracket\right\rangle_{E} \\
& -\sum_{E} \frac{\delta}{2 v}\left\langle\llbracket v \partial_{n} \boldsymbol{u}_{h}-p_{h} \boldsymbol{n} \rrbracket, \llbracket v \partial_{n} \boldsymbol{v}_{h}+q_{h} \boldsymbol{n} \rrbracket\right\rangle_{E}+\sum_{K} \tau^{-1}\left\langle\boldsymbol{u}^{\prime}, \boldsymbol{v}^{\prime}\right\rangle_{K} . \\
& \text { Taking }\left[\boldsymbol{v}_{h}, q_{h}\right]=\left[\boldsymbol{u}_{h}, p_{h}\right] \text { and } \boldsymbol{v}^{\prime}=\boldsymbol{u}^{\prime} \text { it follows that } \\
& B_{\exp }\left(\left[\boldsymbol{u}_{h}, p_{h}\right], \boldsymbol{u}^{\prime} ;\left[\boldsymbol{u}_{h}, p_{h}\right], \boldsymbol{u}^{\prime}\right)=v\left\|\nabla \boldsymbol{u}_{h}\right\|^{2}+\sum_{K}\left\langle\boldsymbol{u}^{\prime},-2 v \Delta \boldsymbol{u}_{h}\right\rangle_{K} \\
& +\sum_{E}\left\langle\left\{\boldsymbol{u}^{\prime}\right\}, \llbracket v \partial_{n} \boldsymbol{u}_{h}+p_{h} \boldsymbol{n} \rrbracket\right\rangle_{E} \\
& \left.-\sum_{E} \frac{\delta}{2} v \| \llbracket \partial_{n} \boldsymbol{u}_{h}\right]\left\|_{E}^{2}+\sum_{E} \frac{\delta}{2 v}\right\| \llbracket n p_{h} \rrbracket \|_{E}^{2}
\end{aligned}
$$

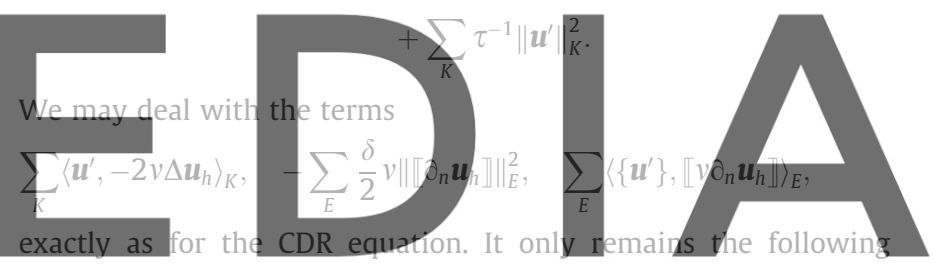

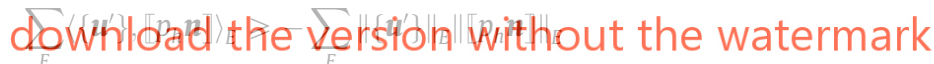

$$
\begin{aligned}
& \geqslant-\sum_{E}\left(\frac{\beta v}{2 \delta}\left\|\left\{\boldsymbol{u}^{\prime}\right\}\right\|_{E}^{2}+\frac{\delta}{2 \beta v}\left\|\llbracket n p_{h} \rrbracket\right\|_{E}^{2}\right) \\
& \geqslant-\sum_{K} \frac{\beta v}{2 \delta}\left\|\boldsymbol{u}^{\prime}\right\|_{\partial K}^{2}-\sum_{E} \frac{\delta}{2 \beta v}\left\|\llbracket \boldsymbol{n} p_{h} \rrbracket\right\|_{E}^{2} \\
& \geqslant-\sum_{K} \frac{\beta v}{2 \delta_{0} h^{2}} C_{\text {tr }}\left\|\boldsymbol{u}^{\prime}\right\|_{K}^{2}-\sum_{E} \frac{\delta}{2 \beta v}\left\|\llbracket \boldsymbol{n} p_{h} \rrbracket\right\|_{E}^{2},
\end{aligned}
$$

which holds for all $\beta>0$. Taking it sufficiently large $(\beta>1)$ and proceeding exactly as for the CDR equation we obtain

Theorem 2. There are constants $C_{1}$ and $\delta_{0}$ in the definition of the stabilization parameters such that

$$
\begin{aligned}
& B_{\exp }\left(\left[\boldsymbol{u}_{h}, p_{h}\right], \boldsymbol{u}^{\prime} ;\left[\boldsymbol{u}_{h}, p_{h}\right], \boldsymbol{u}^{\prime}\right) \\
& \quad \geqslant C\left(v\left\|\nabla \boldsymbol{u}_{h}\right\|^{2}+\sum_{E} \frac{\delta}{v}\left\|\llbracket \boldsymbol{n} p_{h} \rrbracket\right\|_{E}^{2}+\sum_{K} \tau^{-1}\left\|\boldsymbol{u}^{\prime}\right\|_{K}^{2}\right) .
\end{aligned}
$$

Remark 7. In the previous estimate, it is important to note that

- Contrary to the CDR equation, now there is a clear gain by accounting for the subscales on the boundary: we have control on the pressure jumps over interelement boundaries. This in particular stabilizes elements with discontinuous pressures.

- Control over $\left\|\llbracket \boldsymbol{n} p_{h} \rrbracket\right\|_{E}^{2}$ can be transformed into $L^{2}$ control over $p_{h}$. This can be proved for example using the strategy presented in [11] and in references therein.

The stability estimate obtained is clearly optimal. 


\section{Darcy flow}

\subsection{Problem statement and finite element approximation}

We will consider here the simplest situation of Darcy's problem in which the permeability is isotropic and uniform. The problem to be solved consists in finding a velocity $\boldsymbol{u}$ and a pressure $p$ such that $\kappa^{-1} \boldsymbol{u}+\nabla p=\mathbf{0}$ in $\Omega$,

$\nabla \cdot \boldsymbol{u}=f \quad$ in $\Omega$,

$\boldsymbol{u} \cdot \boldsymbol{n}=0 \quad$ on $\partial \Omega$,

where $\kappa$ is the permeability coefficient. The functional spaces where the problem can be posed are

$V=H_{0}(\operatorname{div}, \Omega), Q=L^{2}(\Omega) / \mathbb{R}$,

for the velocity and the pressure, respectively. In this case, $f \in L^{2}(\Omega)$. The classical variational formulation of the Darcy problem is well posed in these spaces. However, it is observed from the momentum equation that in fact the pressure will belong to $H^{1}(\Omega) / \mathbb{R}$.

The weak form of the problem is

$\left(\kappa^{-1} \boldsymbol{u}, \boldsymbol{v}\right)-(p, \nabla \cdot \boldsymbol{v})=0$,

$(q, \nabla \cdot \boldsymbol{u})=(q, f)$

which must hold for all $[\boldsymbol{v}, q] \in V \times Q$.

As in the previous section, the finite element spaces for velocity and pressure will be respectively denoted by $V_{h} \subset V, Q_{h} \subset Q$ (con-

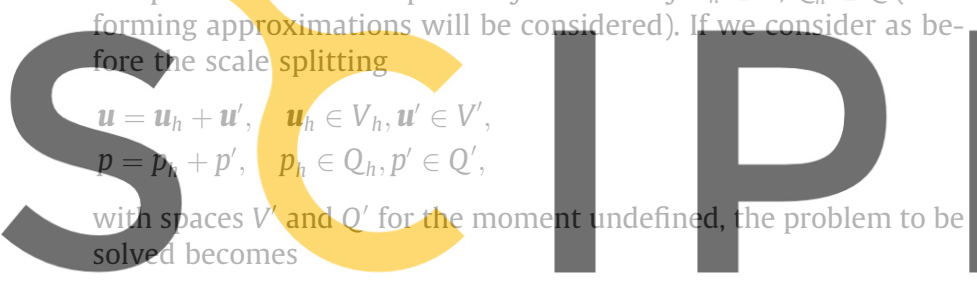

$\left(\kappa^{-1} \boldsymbol{u}_{h}, \boldsymbol{v}_{h}\right)+\left(\kappa^{-1} \boldsymbol{u}^{\prime}, \boldsymbol{v}_{h}\right)-\left(p_{h}, \nabla \cdot \boldsymbol{v}_{h}\right)-\left(p^{\prime}, \nabla \cdot \boldsymbol{v}_{h}\right)=0 \quad \forall \boldsymbol{v}_{h} \in V_{h}$, Register for free at https//www.scipedia.com(to)

$$
\left(q_{h}, \nabla \cdot \boldsymbol{u}_{h}\right)-\sum_{K}\left(\boldsymbol{u}^{\prime}, \nabla q_{h}\right)_{K}+\sum_{K}\left(q_{h}, \boldsymbol{n} \cdot \boldsymbol{u}^{\prime}\right)_{\partial K}=\left(q_{h}, f\right) \quad \forall q_{h} \in Q_{h},
$$

together with the equations obtained by testing the differential equations with the velocity and pressure subscale test functions.

In this case, we need to deal both with a velocity and with a pressure subscale, which makes the derivation of a closed form for them more involved than for the problems of Sections 2 and 3. This can be done in a similar way as for the Stokes problem in [11]. If $P_{V^{\prime}}$ and $P_{Q^{\prime}}$ denote the $L^{2}$-projection onto $V^{\prime}$ and $Q^{\prime}$, respectively, the final result is that $\boldsymbol{u}^{\prime}$ and $p^{\prime}$ can be approximated in the element interiors by

$$
\boldsymbol{u}=-P_{V^{\prime}}\left(\boldsymbol{u}_{h}+\kappa \nabla p_{h}\right)
$$$$
p^{\prime}=\tau_{p} P_{Q^{\prime}}\left(f-\nabla \cdot \boldsymbol{u}_{h}\right),
$$

where the stabilization parameter $\tau_{p}$ is given by

$\tau_{p}=C_{p} \frac{h^{2}}{\kappa}$

$C_{p}$ being an algorithmic constant. The main idea to obtain this approximation is to approximate the Darcy operator in the equation for the subscales by a matrix $\operatorname{diag}\left(\tau_{u}^{-1} \boldsymbol{I}, \tau_{p}^{-1}\right)$, where $\boldsymbol{I}$ is the $d \times d$ identity. Using an approximate Fourier analysis it can be shown that the norm of this matrix is an approximate upper bound to the norm of the Darcy operator if $\tau_{u}=1$ and $\tau_{p}$ is given by (56) (see [11] for details about this approach).
It is convenient to write the previous approximation in 'weak' form as follows:

$\left(\kappa^{-1} \boldsymbol{u}^{\prime}, \boldsymbol{v}^{\prime}\right)+\left(\kappa^{-1} \boldsymbol{u}_{h}, \boldsymbol{v}^{\prime}\right)+\sum_{K}\left(\nabla p_{h}, \boldsymbol{v}^{\prime}\right)=0 \quad \forall \boldsymbol{v}^{\prime} \in V^{\prime}$,

$\left(q^{\prime}, \nabla \cdot \boldsymbol{u}_{h}\right)+\sum_{K} \tau_{p}^{-1}\left(p^{\prime}, q^{\prime}\right) \stackrel{K}{=}\left(q^{\prime}, f\right) \quad \forall q^{\prime} \in Q^{\prime}$.

\subsection{Subscales on the element boundaries}

The transmission conditions for this problem are different from those of the Stokes problem of the previous section. First of all, observe that

- Only the velocity subscale is needed on the boundary of the elements (see (54) and (55)).

- For each element, this velocity subscale can be computed from the pressure subscale on the boundary by projecting the momentum equation.

- Since in fact $p \in H^{1}(\Omega), p$ must be such that

$$
\llbracket \boldsymbol{n} p \rrbracket_{E}=\mathbf{0}, \quad \llbracket \partial_{n} p \rrbracket_{E}=0 .
$$

Eq. (57) are the transmission conditions that have to allow us to compute the subscales on the element boundaries. Since the pressure is allowed to be discontinuous across these interelement boundaries, the pressure subscale must also be allowed to be discontinuous. Let us denote by $p_{h E_{i}}$ the pressure finite element function on an edge $E$ from the side of $K_{i}$ (see again Fig. 1 ) and $p_{E_{i}}^{\prime}$ the
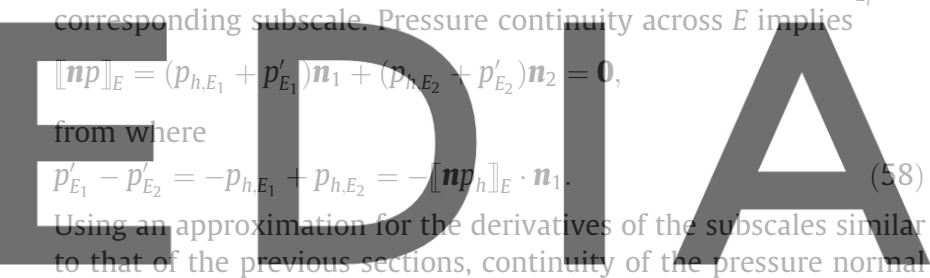

derivative implies:

\section{downtoal that yersion without, the watermark}

from where

$\hat{p}_{E_{1}}^{\prime}+\hat{p}_{E_{2}}^{\prime}=\hat{p}_{K_{1}}^{\prime}+\hat{p}_{K_{2}}^{\prime}-\delta \llbracket \partial_{n} \hat{p}_{h} \rrbracket_{E}$.

The solution of system (58) and (59) yields

$$
p_{\partial K}^{\prime}=\left\{p_{K}^{\prime}\right\}_{\partial K}-\frac{\delta}{2} \llbracket \partial_{n} p_{h} \rrbracket_{\partial K}-\frac{1}{2} \llbracket \boldsymbol{n} p_{h} \rrbracket_{\partial K} \cdot \boldsymbol{n} .
$$

Eq. (60) is the expression of the pressure subscale on the element edges (now discontinuous), obtained from the application of our ideas to the Darcy problem. However, as mentioned earlier, this expression is only required to compute the velocity subscales on the edges, again considering them discontinuous. Projecting the momentum equation on the element boundaries we have:

$$
\begin{aligned}
\left.\boldsymbol{n} \cdot \boldsymbol{u}^{\prime}\right|_{\partial} K= & -\left.\boldsymbol{n} \cdot \boldsymbol{u}_{h}\right|_{\partial} K-\left.\kappa \partial_{n} p_{h}\right|_{\partial} K-\left.\kappa \partial_{n} p^{\prime}\right|_{\partial} K \\
= & -\left.\boldsymbol{n} \cdot \boldsymbol{u}_{h}\right|_{\partial} K-\left.\kappa \partial_{n} p_{h}\right|_{\partial} K-\frac{\kappa}{\delta}\left(p_{\partial}^{\prime} K-p_{K}^{\prime}\right) \\
= & -\left.\boldsymbol{n} \cdot \boldsymbol{u}_{h}\right|_{\partial} K-\left.\kappa \partial_{n} p_{h}\right|_{\partial} K \\
& -\frac{\kappa}{\delta}\left[\left\{p_{K}^{\prime}\right\}_{\partial} K-\frac{\delta}{2} \llbracket \partial_{n} p_{h} \rrbracket_{\partial} K-\frac{1}{2} \llbracket n p_{h} \rrbracket_{\partial} K \cdot \boldsymbol{n}-p_{K}^{\prime}\right] \\
= & -\left.\boldsymbol{n} \cdot \boldsymbol{u}_{h}\right|_{\partial} K-\left.\kappa \partial_{n} p_{h}\right|_{\partial} K+\frac{\kappa}{2} \llbracket \partial_{n} p_{h} \rrbracket_{\partial} K \\
& +\frac{\kappa}{2 \delta} \llbracket \boldsymbol{n}\left(p_{h}+p_{K}^{\prime}\right) \rrbracket_{\partial} K \cdot \boldsymbol{n},
\end{aligned}
$$


from where we obtain the expression for the velocity subscale on $\partial K$ :

$$
\left.\boldsymbol{n} \cdot \boldsymbol{u}^{\prime}\right|_{\partial K}=-\left.\boldsymbol{n} \cdot \boldsymbol{u}_{h}\right|_{\partial K}-\left.\kappa\left\{\partial_{h} p_{h}\right\}\right|_{\partial K}+\frac{\kappa}{2 \delta} \llbracket \boldsymbol{n}\left(p_{h}+p_{K}^{\prime}\right) \rrbracket_{\partial K} \cdot \boldsymbol{n} .
$$

Since no velocity derivatives appear in the transmission conditions for this problem, the velocity subscale on $\partial K$ turns out to be independent from the velocity subscale on $K$.

Note now that all the terms on the RHS of (61) are vectors whose normal component is continuous across interelement boundaries (the first because we assume $V_{h} \subset V$ ). If $\boldsymbol{w}$ is a vector defined on $E$, with continuous normal component, it holds that $\sum_{K}\left\langle q_{h}, \boldsymbol{n} \cdot \boldsymbol{w}\right\rangle_{\partial K}=\sum_{E}\left\langle\llbracket \boldsymbol{n} q_{h} \rrbracket, \boldsymbol{w}\right\rangle_{E}$.

Using this in the finite element approximation for the continuity equation we obtain the final problem to be solved, which consists of finding $\boldsymbol{u}_{h} \in V_{h}, p_{h} \in Q_{h}, \boldsymbol{u}^{\prime} \in V^{\prime}$ and $p^{\prime} \in Q^{\prime}$ such that

$\left(\kappa^{-1} \boldsymbol{u}_{h}, \boldsymbol{v}_{h}\right)+\left(\kappa^{-1} \boldsymbol{u}^{\prime}, \boldsymbol{v}_{h}\right)-\left(p_{h}, \nabla \cdot \boldsymbol{v}_{h}\right)-\left(p^{\prime}, \nabla \cdot \boldsymbol{v}_{h}\right)=0 \quad \forall \boldsymbol{v}_{h} \in V_{h}$,
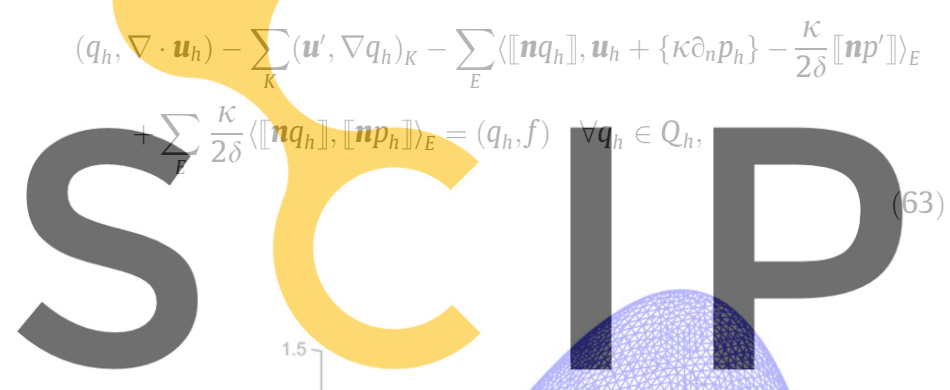

Register for free at https//www.scipedia.com to

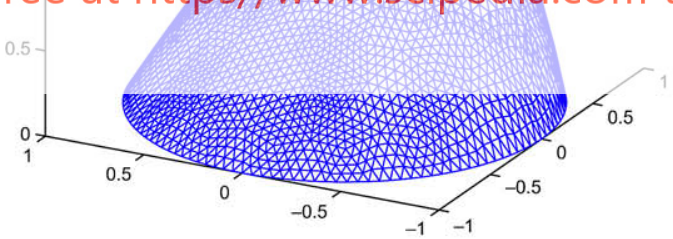

$\left(q^{\prime}, \nabla \cdot \boldsymbol{u}_{h}\right)+\sum_{K} \tau_{p}^{-1}\left(p^{\prime}, q^{\prime}\right)=\left(q^{\prime}, f\right) \quad \forall q^{\prime} \in Q^{\prime}$,

with $\tau_{p}$ given by (56).

\subsection{Stability analysis}

The previous problem can be written as

$B_{\exp }\left(\boldsymbol{u}_{h}, p_{h}, \boldsymbol{u}^{\prime}, p^{\prime} ; \boldsymbol{v}_{h}, q_{h}, \boldsymbol{v}^{\prime}, q^{\prime}\right)=\left(q_{h}, f\right)+\left(q^{\prime}, f\right)$,

with the obvious definition for the bilinear form $B_{\text {exp }}$. The stability analysis in this case is a bit more delicate than for the CDR equation and for the Stokes problem. The problem is that $B_{\exp }$ is not coercive, but satisfies an inf-sup condition in a norm to be introduced in the following.

We assume that the decomposition $V_{h} \oplus V^{\prime}$ is $I^{2}$-stable, in the sense that for any functions $v_{h} \in V_{h}$ and $v^{\prime} \in V^{\prime}$ we have

$$
\left\|\boldsymbol{v}_{h}+\boldsymbol{v}^{\prime}\right\|^{2} \geqslant C_{\mathrm{dec}}\left(\left\|\boldsymbol{v}_{h}\right\|^{2}+\left\|\boldsymbol{v}^{\prime}\right\|^{2}\right) \text {, }
$$

for a constant $C_{\text {dec }}$ independent of the equation parameters and of the mesh size. In general, $C_{\mathrm{dec}} \leqslant 1$ and if $V^{\prime}$ is taken $L^{2}$-orthogonal to $V_{h}, C_{\mathrm{dec}}=1$.

Let $\boldsymbol{U}_{h}=\left[\boldsymbol{u}_{h}, p_{h}, \boldsymbol{u}^{\prime}, p^{\prime}\right]$ be the unknown of the problem and $\boldsymbol{V}_{h}=\left[\boldsymbol{v}_{h}, q_{h}, \boldsymbol{v}^{\prime}, q^{\prime}\right]$ the corresponding vector of test functions. Let also
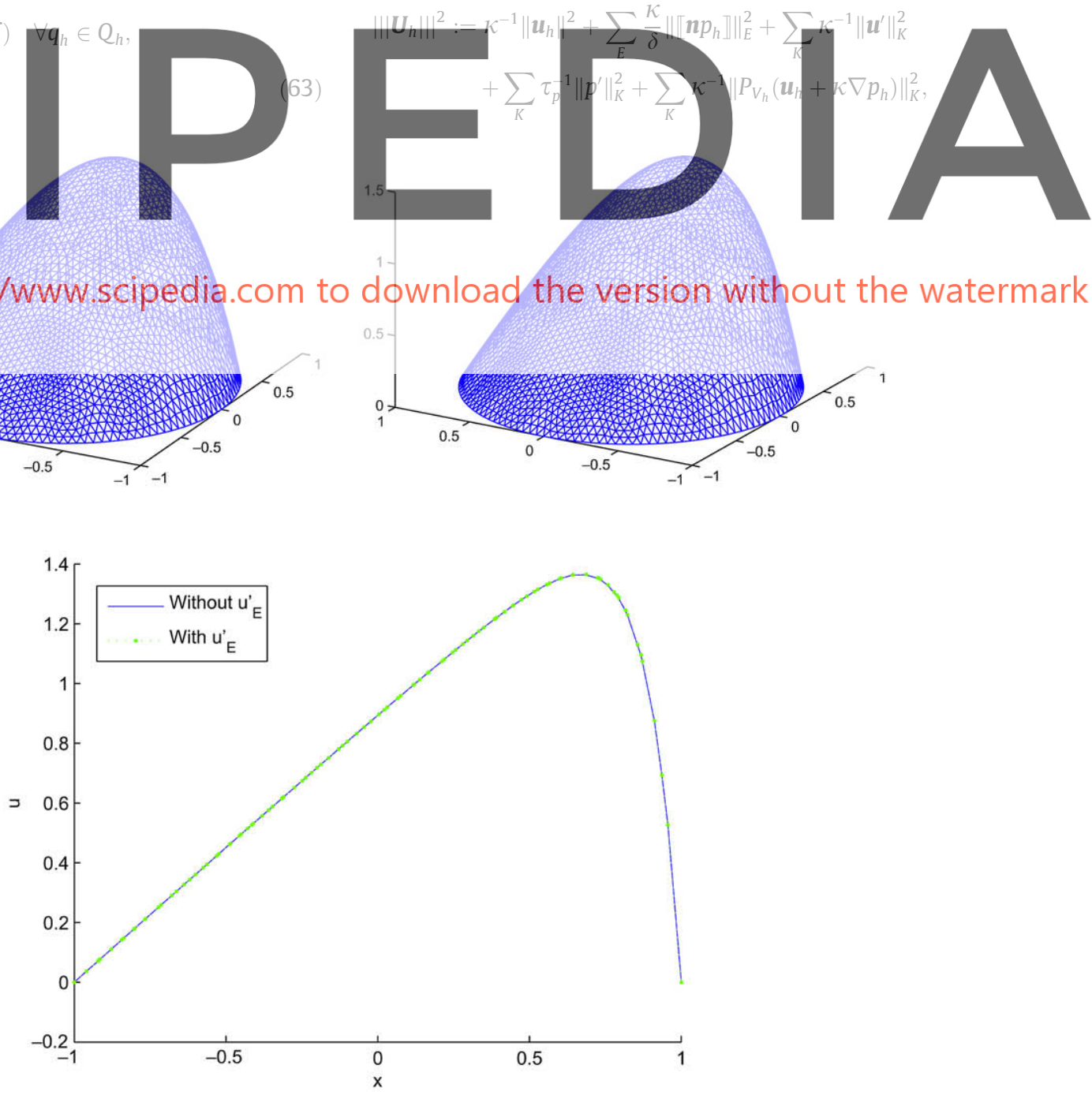

Fig. 2. Elevations for the diffusion dominated problem without (left) and considering (right) $u_{E}^{\prime}$. Cut along $y=0$ (bottom). 
where $P_{V_{h}}$ is the $L^{2}$-projection onto $V_{h}$. However, later on we will introduce another norm in which stability holds and that clearly displays the stability enhancement we obtain with respect to the classical Galerkin method.

Let us start writing

$\boldsymbol{u}_{h}+\kappa \nabla p_{h}=P_{V_{h}}\left(\boldsymbol{u}_{h}+\kappa \nabla p_{h}\right)+P_{V^{\prime}}\left(\boldsymbol{u}_{h}+\kappa \nabla p_{h}\right):=\boldsymbol{m}_{h}-\boldsymbol{u}^{\prime}$,

which allows us to write

$$
\begin{aligned}
B_{\exp }\left(\boldsymbol{U}_{h}, \boldsymbol{U}_{h}\right)= & \kappa^{-1}\left\|\boldsymbol{u}_{h}+\boldsymbol{u}^{\prime}\right\|^{2}+\sum_{E} \frac{\kappa}{2 \delta}\left\|\llbracket \boldsymbol{n} p_{h} \rrbracket\right\|_{E}^{2}+\sum_{K} \tau_{p}^{-1}\left\|p^{\prime}\right\|_{K}^{2} \\
& -\sum_{E}\left\langle\llbracket \boldsymbol{n} p_{h} \rrbracket, \boldsymbol{m}_{h}\right\rangle_{E}+\sum_{E}\left\langle\llbracket \boldsymbol{n} p_{h} \rrbracket,\left\{\boldsymbol{u}^{\prime}\right\}\right\rangle_{E} \\
& +\sum_{E} \frac{\kappa}{2 \delta}\left\langle\llbracket \boldsymbol{n} p_{h} \rrbracket, \llbracket \boldsymbol{n} p^{\prime} \rrbracket\right\rangle_{E} .
\end{aligned}
$$

The objective now is to bound the last three terms in the RHS of this equality. Let us start with the last one. Using (46) we have that

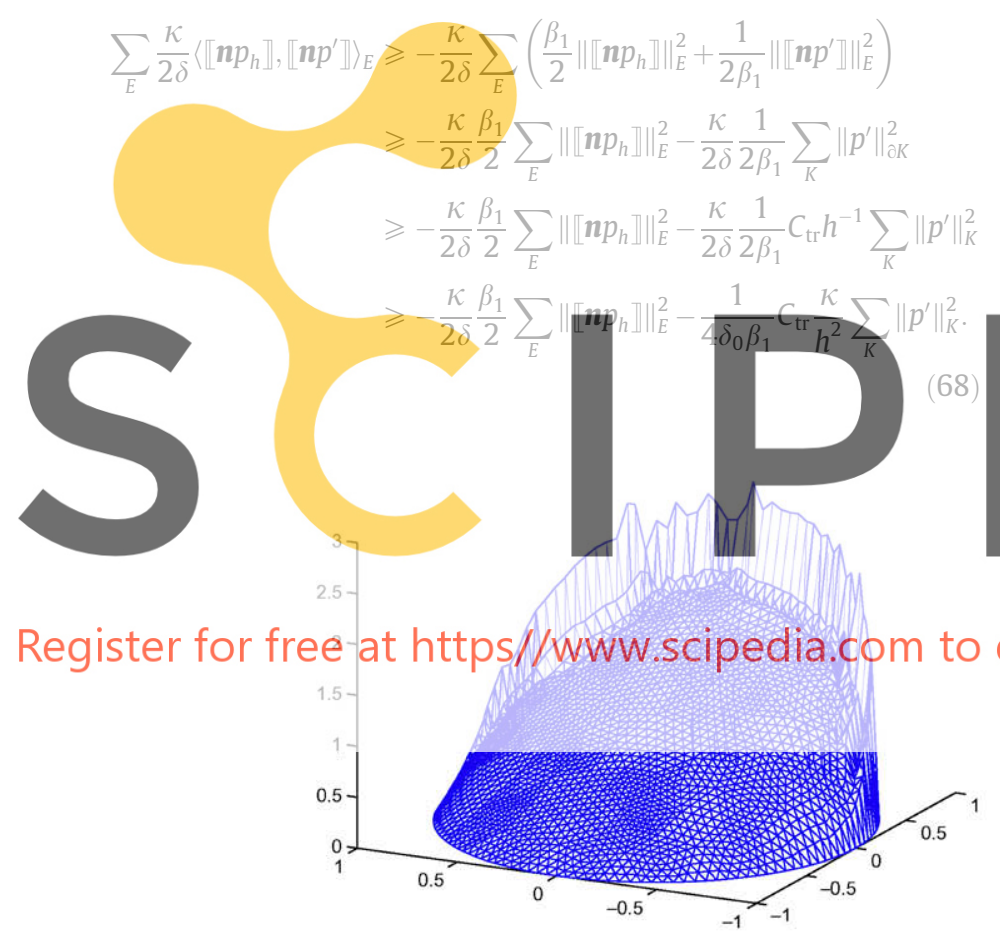

68

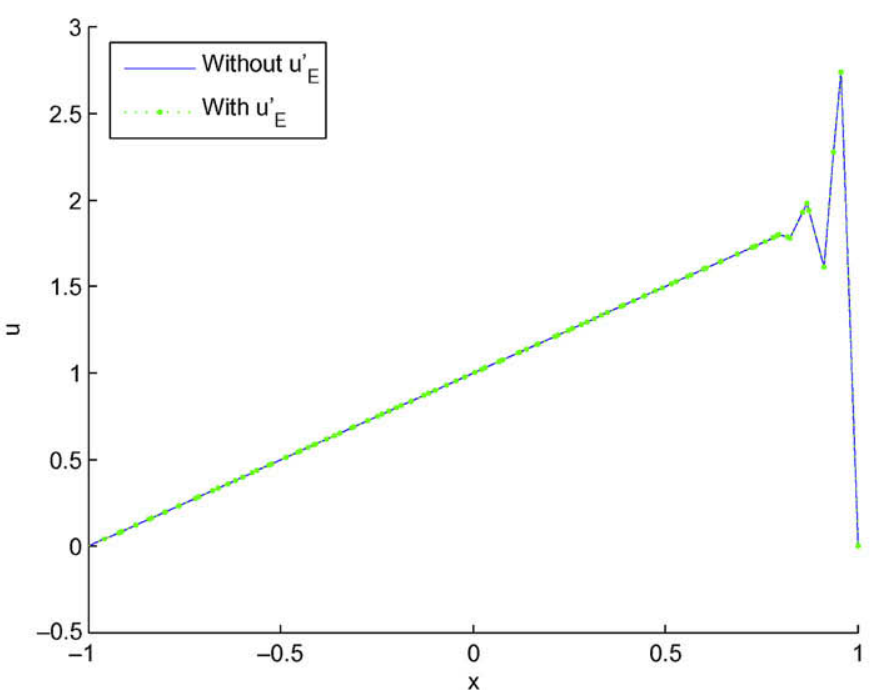

Fig. 3. Elevations for the convection dominated problem without (left) and considering (right) $u_{E}^{\prime}$. Cut along $y=0$ (bottom)

Using (66), (68) and (69) in (67) we have

$B_{\exp }\left(\boldsymbol{U}_{h},\left[\boldsymbol{m}_{h}, 0, \mathbf{0}, 0\right]\right)$$$
=\kappa^{-1}\left(\boldsymbol{m}_{h}, \boldsymbol{m}_{h}\right)-\left(p^{\prime}, \nabla \cdot \boldsymbol{m}_{h}\right)-\sum_{E}\left\langle\llbracket \boldsymbol{n} p_{h} \rrbracket, \boldsymbol{m}_{h}\right\rangle_{E}
$$

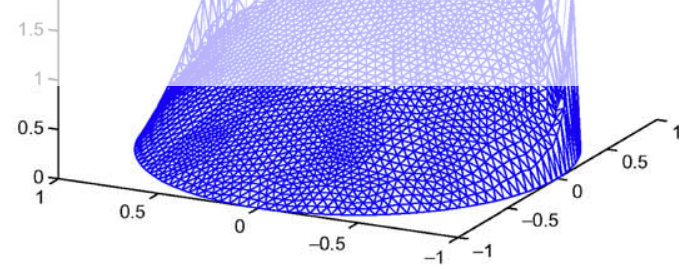

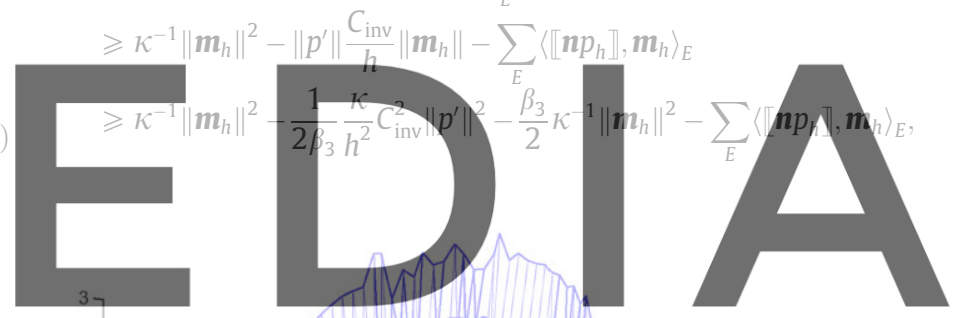

$$
\begin{aligned}
& \text { We also have that } \\
& \begin{aligned}
\sum_{E}\left\langle\llbracket \boldsymbol{n} p_{h} \rrbracket,\left\{\boldsymbol{u}^{\prime}\right\}\right\rangle_{E} & \geqslant-\sum_{E}\left(\frac{\delta}{2 \beta_{2}} \kappa^{-1}\left\|\left\{\boldsymbol{u}^{\prime}\right\}\right\|_{E}^{2}+\frac{\beta_{2}}{2 \delta} \kappa\left\|\llbracket \boldsymbol{n} p_{h} \rrbracket\right\|_{E}^{2}\right) \\
& \geqslant-\sum_{K} \frac{\delta}{2 \beta_{2}} \kappa^{-1}\left\|\boldsymbol{u}^{\prime}\right\|_{\partial K}^{2}-\sum_{E} \frac{\beta_{2}}{2 \delta} \kappa\left\|\llbracket \boldsymbol{n} p_{h} \rrbracket\right\|_{E}^{2} \\
& \geqslant-\sum_{K} \frac{\delta_{0}}{2 \beta_{2}} C_{\mathrm{tr}} \kappa^{-1}\left\|\boldsymbol{u}^{\prime}\right\|_{K}^{2}-\sum_{E} \frac{\beta_{2}}{2 \delta} \kappa\left\|\llbracket \boldsymbol{n} p_{h} \rrbracket\right\|_{E}^{2}
\end{aligned}
\end{aligned}
$$

$$
\begin{aligned}
B_{\exp }\left(\boldsymbol{U}_{h}, \boldsymbol{U}_{h}\right) \geqslant & \kappa^{-1} C_{\mathrm{dec}}\left\|\boldsymbol{u}_{h}\right\|^{2}+\kappa^{-1}\left(C_{\mathrm{dec}}-\frac{\delta_{0}}{2 \beta_{2}} C_{\mathrm{tr}}\right) \sum_{K}\left\|\boldsymbol{u}^{\prime}\right\|_{K}^{2} \\
& +\sum_{E} \frac{\kappa}{2 \delta}\left(1-\frac{\beta_{1}}{2}-\beta_{2}\right)\left\|\llbracket \boldsymbol{n} p_{h} \rrbracket\right\|_{E}^{2} \\
& +\sum_{K} \frac{\kappa}{h^{2}}\left(\frac{1}{C_{p}}-\frac{C_{\mathrm{tr}}}{4 \delta_{0} \beta_{1}}\right)\left\|p^{\prime}\right\|_{K}^{2}-\sum_{E}\left\langle\llbracket \boldsymbol{n} p_{h} \rrbracket, \boldsymbol{m}_{h}\right\rangle_{E} .
\end{aligned}
$$

It remains to control the last term. It is responsible for the fact that the bilinear form $B_{\exp }$ is not coercive, but it only satisfies an inf-sup condition. By the definition of $\boldsymbol{m}_{h}$ and using (45) we have that

$$
=\left(\kappa^{-1} \boldsymbol{u}_{h}+\nabla p_{h}, \boldsymbol{m}_{h}\right)+\left(\kappa^{-1} \boldsymbol{u}^{\prime}, \boldsymbol{m}_{h}\right)-\left(p^{\prime}, \nabla \cdot \boldsymbol{m}_{h}\right)-\sum_{K}\left\langle p_{h}, \boldsymbol{n} \cdot \boldsymbol{m}_{h}\right\rangle_{\curvearrowright K}
$$

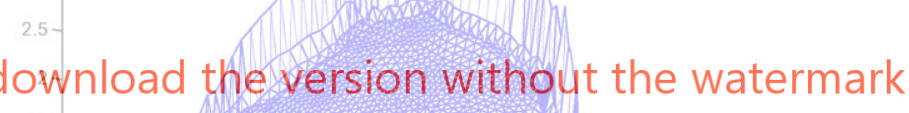


which combined with (70) yields

$$
\begin{aligned}
& B_{\exp }\left(\boldsymbol{U}_{h}, \boldsymbol{U}_{h}+\left[\boldsymbol{m}_{h}, 0, \mathbf{0}, 0\right]\right) \\
& \geqslant \kappa^{-1} C_{\mathrm{dec}}\left\|\boldsymbol{u}_{h}\right\|^{2}+\kappa^{-1}\left(C_{\mathrm{dec}}-\frac{\delta_{0}}{2 \beta_{2}} C_{\mathrm{tr}}\right)\left\|\boldsymbol{u}^{\prime}\right\|^{2} \\
& \quad+\sum_{E} \frac{\kappa}{2 \delta}\left(1-\frac{\beta_{1}}{2}-\beta_{2}\right)\left\|\llbracket \boldsymbol{n} p_{h} \rrbracket\right\|_{E}^{2} \\
& \quad+\sum_{K} \frac{\kappa}{h^{2}}\left(\frac{1}{C_{p}}-\frac{C_{\mathrm{tr}}}{4 \delta_{0} \beta_{1}}-\frac{C_{\mathrm{inv}}^{2}}{2 \beta_{3}}\right)\left\|p^{\prime}\right\|_{K}^{2} \\
& \quad+\kappa^{-1}\left(1-\frac{\beta_{3}}{2}\right)\left\|\boldsymbol{m}_{h}\right\|^{2}-2 \sum_{E}\left\langle\llbracket \boldsymbol{n} p_{h} \rrbracket, \boldsymbol{m}_{h}\right\rangle_{E} .
\end{aligned}
$$

On the other hand

$$
\begin{aligned}
-2 \sum_{E}\left\langle\llbracket \boldsymbol{n} p_{h} \rrbracket, \boldsymbol{m}_{h}\right\rangle_{E} & \geqslant-\sum_{E} \beta_{4} \frac{\kappa}{2 \delta}\left\|\llbracket \boldsymbol{n} p_{h} \rrbracket\right\|_{E}^{2}-\sum_{E} \frac{1}{\beta_{4}} \frac{2 \delta}{\kappa}\left\|\boldsymbol{m}_{h}\right\|_{E}^{2} \\
& \geqslant-\sum_{E} \beta_{4} \frac{\kappa}{2 \delta}\left\|\llbracket \boldsymbol{n} p_{h} \rrbracket\right\|_{E}^{2}-\sum_{K} \frac{1}{\beta_{4}} \kappa^{-1} \delta_{0} C_{\mathrm{tr}}\left\|\boldsymbol{m}_{h}\right\|_{K}^{2},
\end{aligned}
$$

which used in (71) gives
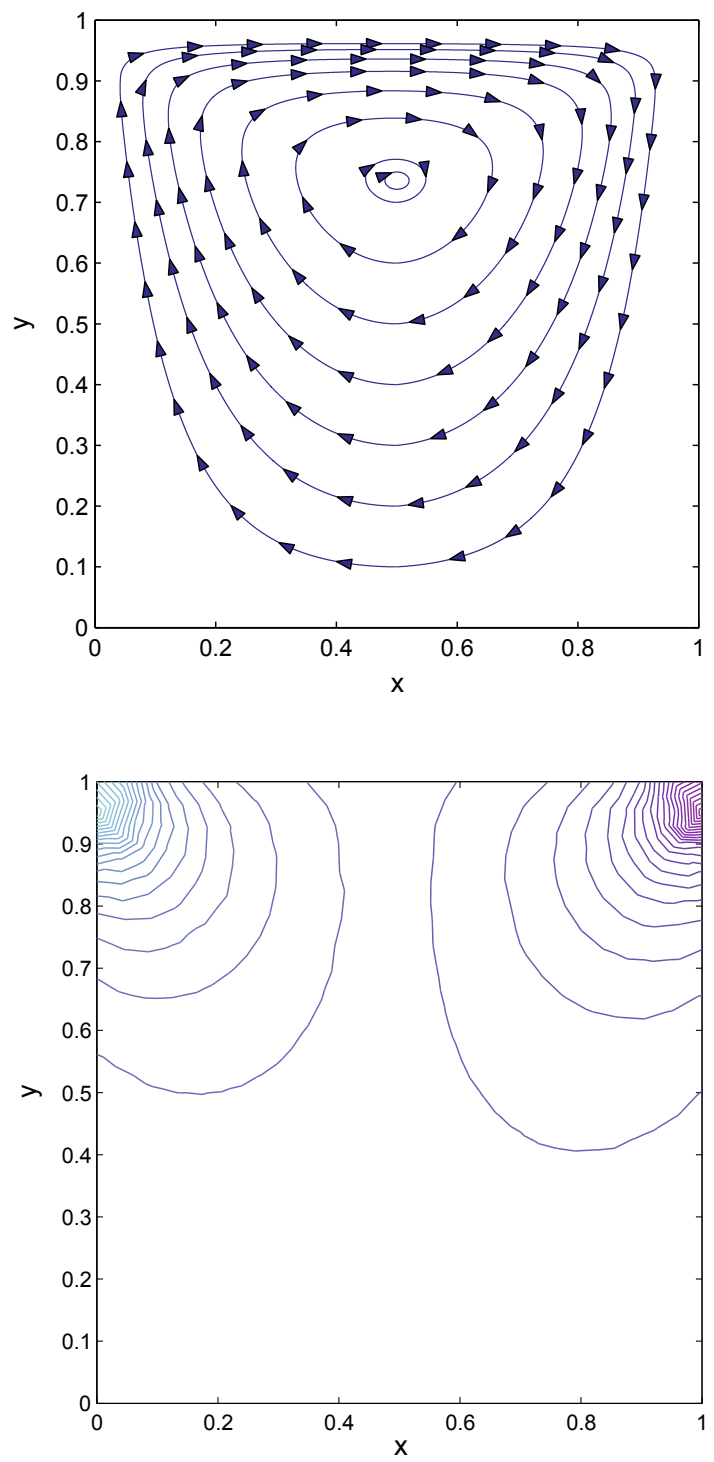

$$
\begin{aligned}
B_{\exp }\left(\boldsymbol{U}_{h}, \boldsymbol{U}_{h}+\left[\boldsymbol{m}_{h}, 0, \mathbf{0}, 0\right]\right) \geqslant & \kappa^{-1} C_{\mathrm{dec}}\left\|\boldsymbol{u}_{h}\right\|^{2}+\kappa^{-1}\left(C_{\mathrm{dec}}-\frac{\delta_{0}}{2 \beta_{2}} C_{\mathrm{tr}}\right)\left\|\boldsymbol{u}^{\prime}\right\|^{2} \\
& +\sum_{E} \frac{\kappa}{2 \delta}\left(1-\frac{\beta_{1}}{2}-\beta_{2}-\beta_{4}\right)\left\|\llbracket \boldsymbol{n} p_{h} \rrbracket\right\|_{E}^{2} \\
& +\sum_{K} \frac{\kappa}{h^{2}}\left(\frac{1}{C_{p}}-\frac{C_{\mathrm{tr}}}{4 \delta_{0} \beta_{1}}-\frac{C_{\mathrm{inv}}^{2}}{2 \beta_{3}}\right)\left\|p^{\prime}\right\|_{K}^{2} \\
& +\kappa^{-1}\left(1-\frac{\beta_{3}}{2}-\frac{\delta_{0} C_{\mathrm{tr}}}{\beta_{4}}\right)\left\|\boldsymbol{m}_{h}\right\|^{2} .
\end{aligned}
$$

From this expression we see that if we take $\beta_{i}, i=1,2,3,4$, sufficiently small, then there exists a constant $C$ for which

$B_{\exp }\left(\boldsymbol{U}_{h}, \boldsymbol{U}_{h}+\left[\boldsymbol{m}_{h}, 0, \mathbf{0}, 0\right]\right) \geqslant C\left|\left\|\boldsymbol{U}_{h}\right\|\right|^{2}$,

provided the constants $\delta_{0}$ and $C_{p}$ are small enough. On the other hand, ||$\left[\boldsymbol{m}_{h}, 0, \mathbf{0}, 0\right]\left|\left\|\leqslant C\left|\left\|\boldsymbol{U}_{h}\right\|\right|\right.\right.$, from where we obtain the result we wished to prove:

Theorem 3. There are constants $C_{p}$ and $\delta_{0}$ in the definition of the stabilization parameters such that for all $\boldsymbol{U}_{h}$ there exists $\boldsymbol{V}_{h}$ such that
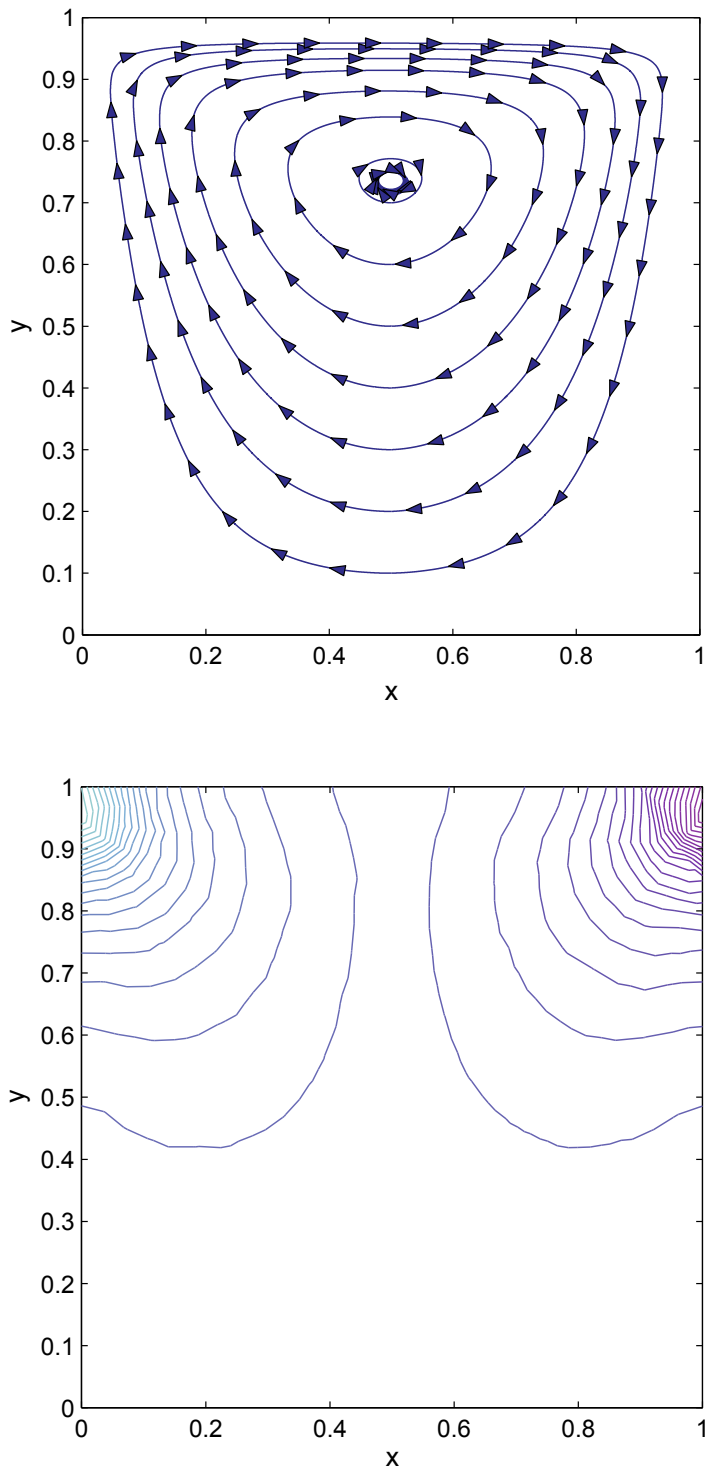

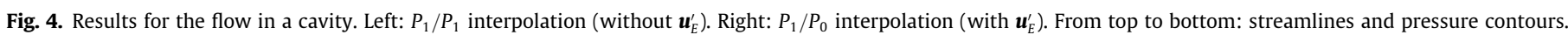


$B_{\exp }\left(\boldsymbol{U}_{h}, \boldsymbol{V}_{h}\right) \geqslant C||\left|\boldsymbol{U}_{h}\right|||||\left|\boldsymbol{V}_{h}\right|||$.

Remark 8. Since $\boldsymbol{u}^{\prime}=P_{V^{\prime}}\left(\boldsymbol{u}_{h}+\kappa \nabla p_{h}\right)$ and in view of (66) our result also applies with the norm

$\left\|\boldsymbol{U}_{h}\right\|\left\|_{*}^{2}:=\kappa^{-1}\right\| \boldsymbol{u}_{h}\left\|^{2}+\kappa \sum_{K}\right\| \nabla p_{h}\left\|_{K}^{2}+\sum_{E} \frac{\kappa}{\delta}\right\| \llbracket \boldsymbol{n} p_{h} \rrbracket\left\|_{E}^{2}+\sum_{K} \tau_{p}^{-1}\right\| p^{\prime} \|_{K}^{2}$,

which allows us to see that the stability result of Theorem 3 is optimal. Moreover, from the expression of $p^{\prime}$ in the element interiors, usually proportional to the velocity divergence, it is possible to control $\left\|\nabla \cdot \boldsymbol{u}_{h}\right\|$ which, together with the stability obtained on $\left\|\boldsymbol{u}_{h}\right\|$, leads to full control of $\boldsymbol{u}_{h}$ in $H_{0}(\operatorname{div}, \Omega)$ (see [3] for further details).

\section{Numerical examples}

In this section, we present the results of some numerical examples in order to study the performance of the presented method. We compare the results obtained using the approximation of the subscales on the interelement boundaries $u_{E}^{\prime}$ given by (36) (or (51) in the case of the Stokes problem) with those obtained considering $u_{E}^{\prime}=0$. A parameter $\delta_{0}=0.2$ has been adopted for the computation of the terms corresponding to the subscales on the element boundaries, as it has proved to be suitable for these numerical examples, even though for the Stokes problem the effect of the choice of $\delta_{0}$ has also been analyzed.

No results for the Darcy problem have been included, since in the case of interest, that is to say, for discontinuous pressure interpolations, the accuracy heavily relies on the expression of the subscales in the element interiors. A thorough discussion and a full convergence analysis can be found in [3].

\subsection{Convection-diffusion equation}

Let us start solving the convection-diffusion equation. We consider a domain $\Omega$ enclosed in a circle of radius $R=1$, which we discretize in a triangular finite element mesh, and we prescribe $u=0 \quad$ on $\partial \Omega$.

We now study two different cases: in the first one diffusion dominates over convection $(k=0.1, \boldsymbol{a}=(1,0), s=0, f=1$ in (1)), while the second one is convection dominated $\left(k=10^{-12}, \boldsymbol{a}=(1,0), s=0\right.$, $f=1$ in (1)). In both the diffusion and the convection dominated cases, no difference between the solution obtained considering $u_{E}^{\prime}$ and the one obtained without considering it can be appreciated. Fig. 2 shows and compares the obtained solution $u$ for the considered methods in the diffusion dominated case, while Fig. 3 does
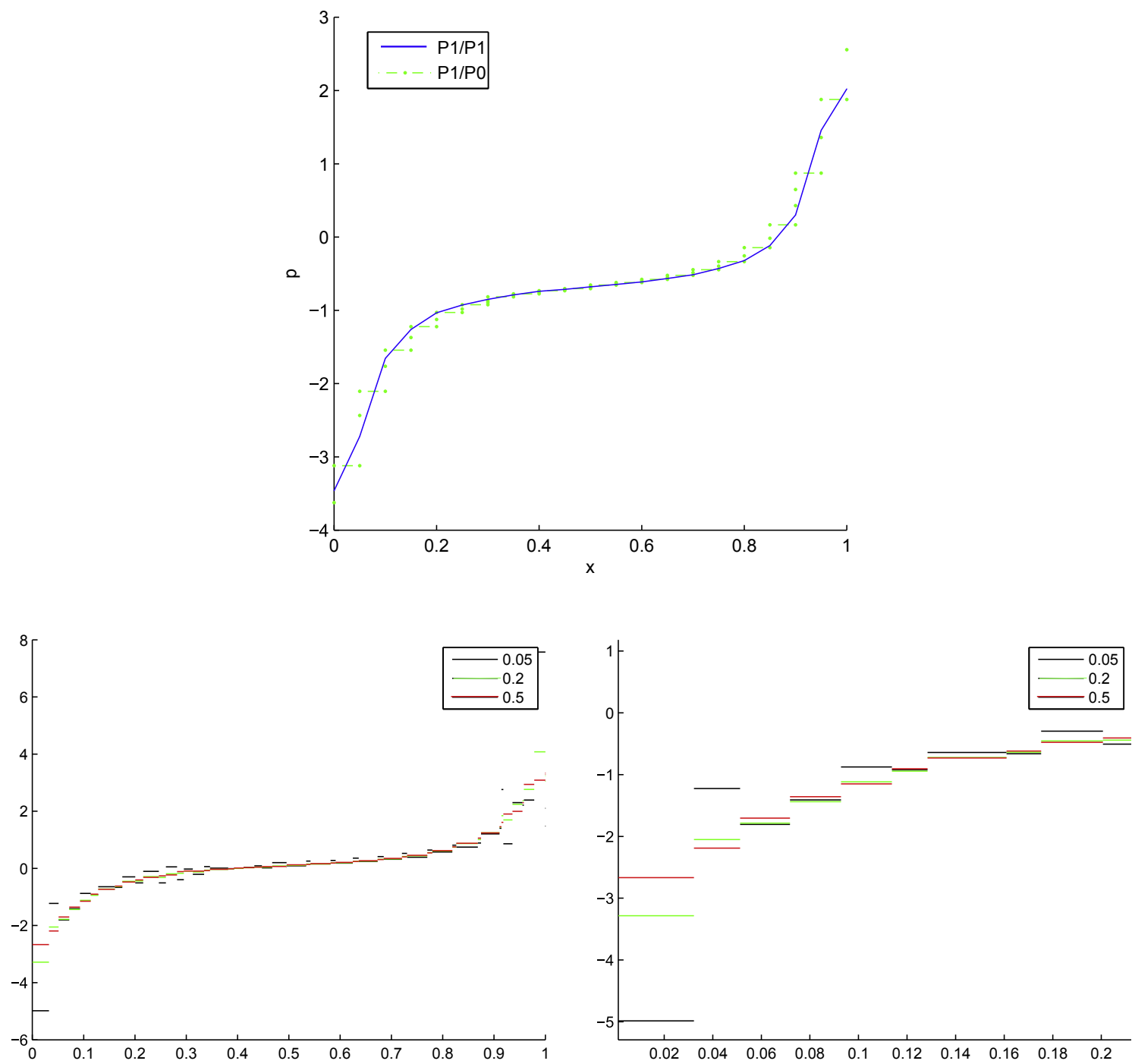

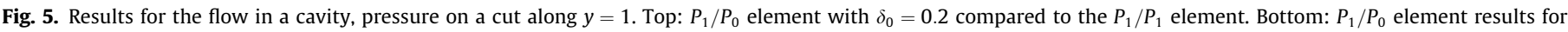
different values of $\delta_{0}$, global cut (left) and detail (right). 

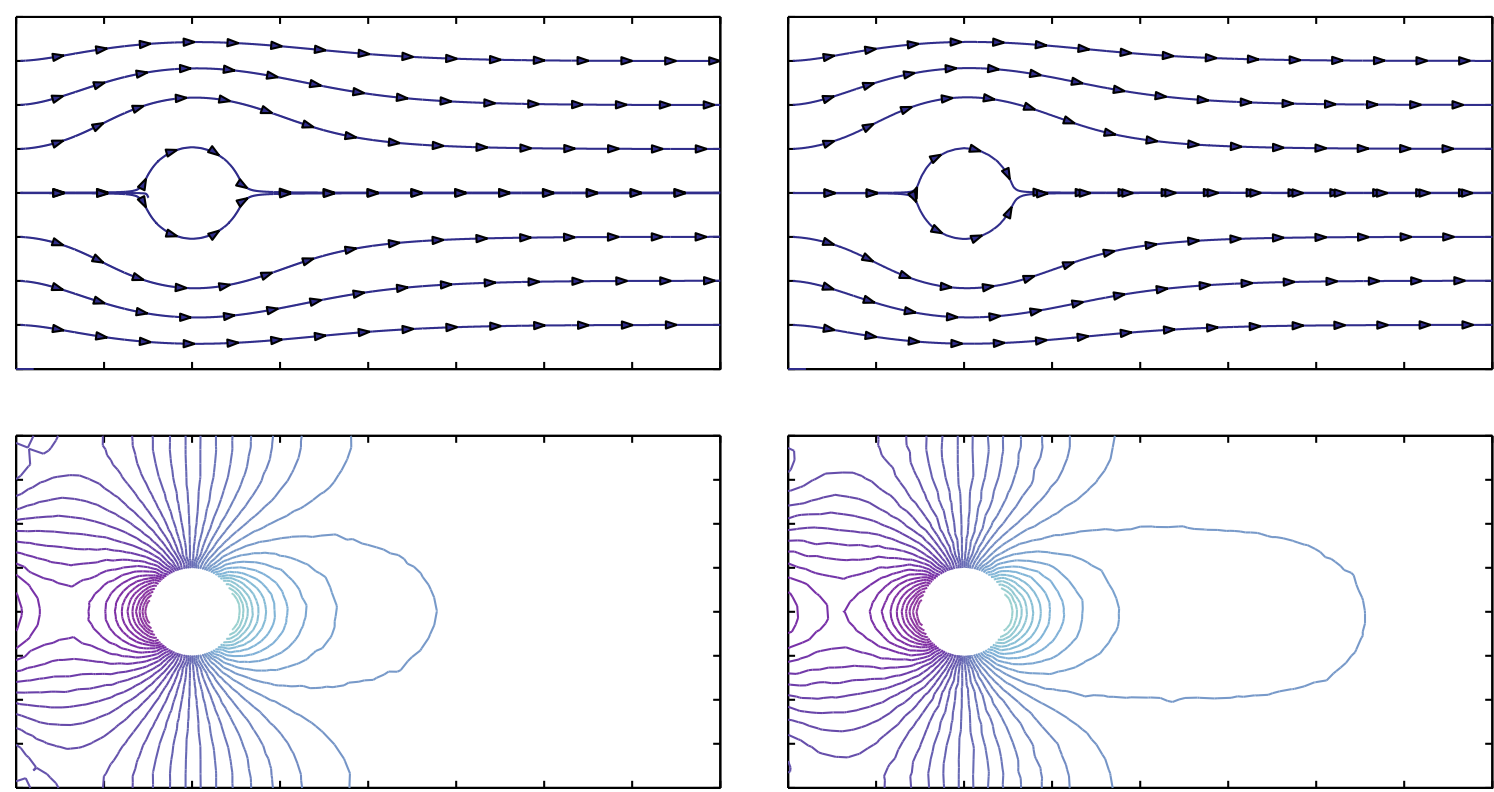

Fig. 6. Results for the flow over a cylinder. Left: $P_{1} / P_{1}$ (without $\boldsymbol{u}_{E}^{\prime}$ ). Right: $P_{1} / P_{0}$ (with $\boldsymbol{u}_{E}^{\prime}$ ). From top to bottom: streamlines and pressure contours.
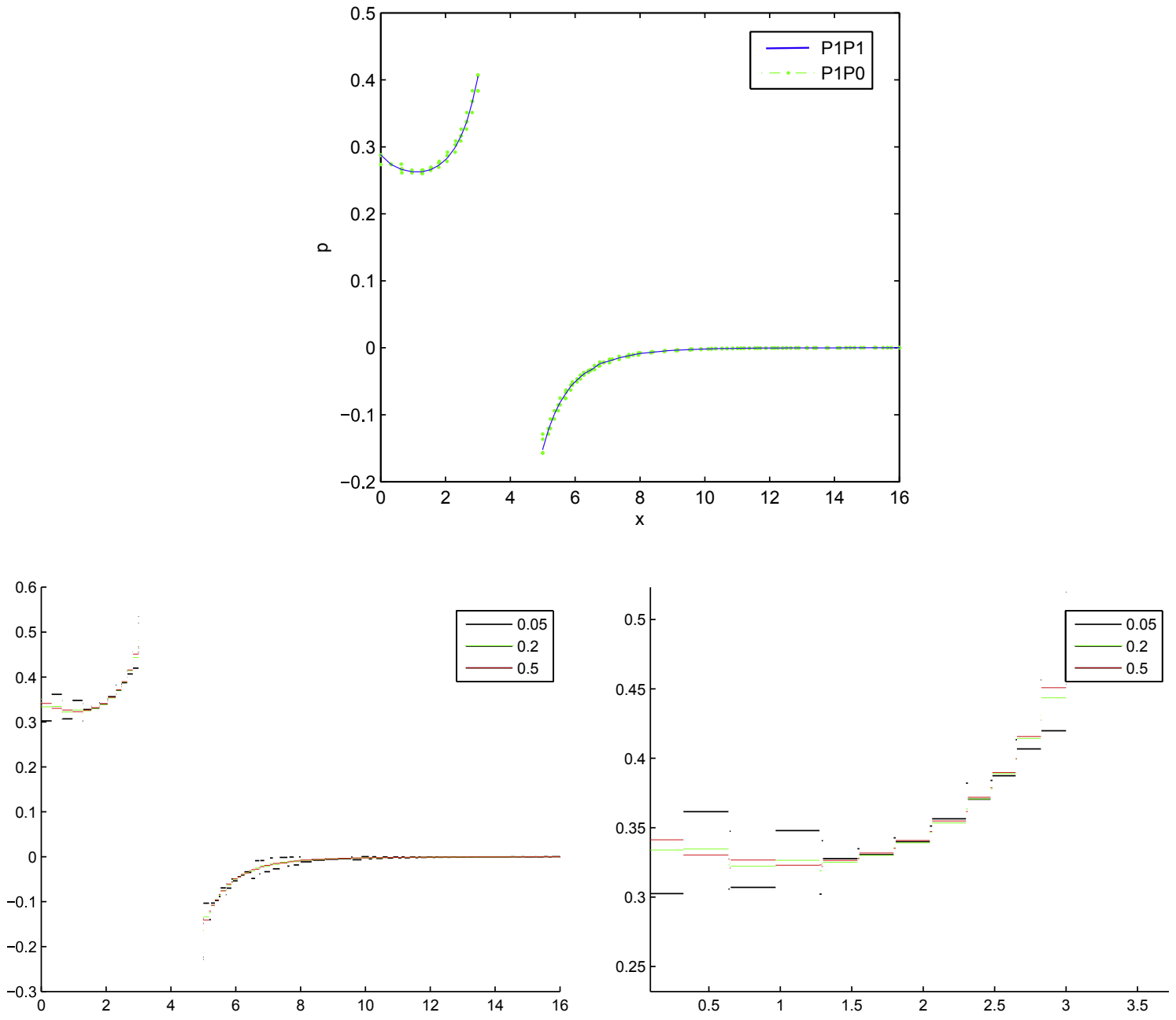

Fig. 7. Results for the flow over a cylinder, pressure in a cut along $y=4$. Top: $P_{1} / P_{0}$ element with $\delta_{0}=0.2$ compared to the $P_{1} / P_{1}$ element. Bottom: $P_{1} / P_{0}$ element results for different values of $\delta_{0}$, global cut (left) and detail (right). 
so when convection dominates over diffusion. In any case, there is no noticeable influence of the value of $\delta_{0}$ on the results.

\subsection{Stokes problem}

In this section, we study the performance of the method proposed for the Stokes problem. As stated in Section 3, considering the contribution of the subscales in the element boundaries $\boldsymbol{u}_{E}^{\prime}$ stabilizes elements with discontinuous pressures. In particular it allows the use of $P_{1} / P_{0}$ (linear-constant) velocity-pressure pairs. Results using $P_{1} / P_{0}$ interpolation and considering the contribution of the subscales on the boundary will be compared with those obtained using $P_{1} / P_{1}$ (linear-linear) velocity-pressure pairs, in which no subscales on the boundaries are considered.

\subsubsection{Flow in a cavity}

In this example, the motion of a fluid enclosed in a square cavity $\Omega=[0,1] \times[0,1]$ is analyzed. The velocity is set to $(1,0)$ at the top horizontal wall $(y=1)$, while it is prescribed to $\mathbf{0}$ on the other walls $(y=0, x=0$ and $x=1)$. Pressure is fixed to 0 in an arbitrary point of the domain.

As Fig. 4 shows, little difference can be observed between results obtained using $P_{1} / P_{1}$ interpolation and those obtained using $P_{1} / P_{0}$ and taking into account the contribution of the subscales on the element boundaries with $\delta_{0}=0.2$. The slight differences which can be observed between both results are due to the fact that a poorer interpolation space for the pressure is used in the second case.

In order to check the behavior of the solution in terms of $\delta_{0}$, Fig. 5 shows a comparison between the pressure along $y=1$ for $\delta_{0}=0.05,0.2$ and 0.5 . Note that this last value would be the maximum allowed by our way to motivate the subscales on the element boundaries (see Fig. 1 ). It is observed that $\delta_{0}=0.05$ allows for pressure oscillations, whereas no much difference is observed for $\delta_{0}=0.2$ and $\delta_{0}=0.5$ (in fact, similar results are obtained for any $\delta_{0}$ greater than 0.1 ). Of course, results are more diffusive the greater the value of $\delta_{0}$ is.

\subsubsection{Flow over a cylinder}

In this example, we study the Stokes flow past a cylinder. The computational domain is $\Omega=[0,16] \times[0,8] \backslash D$, with the cylinder $D$ of diameter 2 and centered at $(4,4)$. The velocity at $x=0$ is prescribed at $(1,0)$, whereas at $y=0$ and $y=8$, the $y$-velocity component is prescribed to 0 and the $x$-component is left free. The outflow, where both the $x$ - and $y$ - component are free, is $x=16$. Tractions are set to 0 on the outflow.

As in the previous example, little difference can be appreciated between the solutions obtained with the $P_{1} / P_{1}$ pair with no subscales on the boundaries and the $P_{1} / P_{0}$ element with subscales on the boundaries (see Fig. 6).

Once again, the behavior of the solution in terms of $\delta_{0}$ has been checked. A comparison between the pressure in a cut along $y=4$ is shown in Fig. 7 for $\delta_{0}=0.05,0.2$ and 0.5. The same conclusions as for the cavity flow example can be drawn in this case, namely, $\delta_{0}=0.05$ allows for pressure oscillations which do not appear using $\delta_{0}=0.2$ and $\delta_{0}=0.5$, the latter being more diffusive than the former.

\section{Conclusions}

In this paper, we have extended the two-scale approximation of variational problems with an additional ingredient in the approximation of the subscales, which is an approximation for their values on the interelement boundaries.

The key idea is to assume that the subscales are already computed in the element interiors and to compute the boundary values by imposing the correct transmission conditions of the problem under consideration. Three examples of how to undertake this process have been presented, namely, the CDR equation, the Stokes problem and Darcy's equations.

In order to be as general as possible, examples of how to compute the subscale on the element interiors have been proposed, but not used, in the sense that our developments are applicable to any approximation of these unknowns (provided they satisfy some conditions on the algorithmic constants on which they depend). In fact, we have proved stability estimates for the three problems considered which are valid for any choice of subscales in the interior of the elements. However, convergence analyses, not presented here, require the expressions of these subscales.

For the case of the CDR equation, the new terms introduced by accounting for the subscales on the interelement boundaries do not contribute to stability. However, our analysis and the numerical example presented show that they do not spoil it, and also that accuracy seems also to be unaffected. However, for the Stokes problem and for Darcy flow the terms introduced by the subscales on the boundaries are crucial to provide stability when discontinuous pressure interpolations are used. The stabilizing terms introduced are shared with other formulations that can be found in the literature. However, some non-standard terms also appear. Again, our analysis, and the numerical examples in the case of the Stokes problems, show that these terms do not harm stability.

\section{References}

[1] R. Araya, G. Barrenechea, F. Valentin, Stabilized finite element methods based on multiscale enrichment for the Stokes problem, SIAM J. Numer. Analysis 44 (2006) 322-348.

[2] D.N. Arnold, An interior penalty finite element method with discontinuous elements, SIAM J. Numer. Analysis 19 (1982) 742-760.

[3] S. Badia, R. Codina, Unified stabilized finite element formulations for the Stokes and the Darcy problems, UPCommons, submitted for publication, <http:// hdl.handle.net/2117/2168>

[4] C. Baiocchi, F. Brezzi, L.P. Franca, Virtual bubbles and Galerkin/least-squares type methods (Ga.L.S), Comput. Methods Appl. Mech. Engrg. 105 (1993) 125141.

[5] S.C. Brenner, L.R. Scott, The Mathematical Theory of Finite Element Methods, Springer-Verlag, 1994.

[6] E. Burman, M.A. Fernández, P. Hansbo, Continuous interior penalty finite element method for Oseen's equations, SIAM J. Numer. Analysis 44 (2006) 1248-1274.

[7] E. Burman, P. Hansbo, Edge stabilization for Galerkin approximations of convection-diffusion-reaction problems, Comput. Methods Appl. Mech. Engrg. 193 (2004) 1437-1453.

[8] R. Codina, Comparison of some finite element methods for solving the diffusion convection reaction equation, Comput. Methods Appl. Mech. Engrg. 156 (1998) $185-210$

[9] R. Codina, Stabilization of incompressibility and convection through orthogonal sub-scales in finite element methods, Comput. Methods Appl. Mech. Engrg. 190 (2000) 1579-1599.

[10] R. Codina, Stabilized finite element approximation of transient incompressible flows using orthogonal subscales, Comput. Methods Appl. Mech. Engrg. 191 (2002) 4295-4321.

[11] R. Codina, Finite element approximation of the three field formulation of the Stokes problem using arbitrary interpolations, SIAM J. Numer. Analysis, in press.

[12] R. Codina, J. Principe, O. Guasch, S. Badia, Time dependent subscales in the stabilized finite element approximation of incompressible flow problems, Comput. Methods Appl. Mech. Engrg. 196 (2007) 2413-2430.

[13] A. Ern, J.-L. Guermond, Theory and Practice of Finite Elements, Springer-Verlag, 2004.

[14] L.P. Franca, C. Farhat, Bubble functions prompt unusual stabilized finite element methods, Comput. Methods Appl. Mech. Engrg. 123 (1994) 299-308.

[15] A. Hansbo, P. Hansbo, An unfitted finite element method, based on Nitsche's method, for elliptic interface problems, Comput. Methods Appl. Mech. Engrg. 191 (2002) 5537-5552.

[16] T.J.R. Hughes, Multiscale phenomena: Green's function, the Dirichlet-toNeumann formulation, subgrid scale models, bubbles and the origins of stabilized formulations, Comput. Methods Appl. Mech. Engrg. 127 (1995) 387401.

[17] T.J.R. Hughes, G.R. Feijóo, L. Mazzei, J.B. Quincy, The variational multiscale method - a paradigm for computational mechanics, Comput. Methods Appl. Mech. Engrg. 166 (1998) 3-24. 
[18] T.J.R. Hughes, L.P. Franca, G.M. Hulbert, A new finite element formulation for computational fluid dynamics: VII. The Stokes problem with various wellposed boundary conditions: symmetric formulations that converge for all velocity/pressure spaces, Comput. Methods Appl. Mech. Engrg. 65 (1987) 8596.

[19] T.J.R. Hughes, A. Masud, J. Wan, A stabilized mixed discontinuous Galerkin method for Darcy flow, Comput. Methods Appl. Mech. Engrg. 195 (2006) 3347-3381.

[20] N. Kechkar, D. Silvester, Analysis of locally stabilized mixed finite element methods for the Stokes problem, Math. Comput. 58 (1992) 1-10.

[21] A. Masud, T.J.R. Hughes, A stabilized mixed finite element method for Darcy flow, Comput. Methods Appl. Mech. Engrg. 191 (2002) 4341-4370.
[22] K.B. Nakshatrala, D.Z. Turner, K.D. Hjelmstad, A. Masud, A stabilized mixed finite element method for Darcy flow based on a multiscale decomposition of the solution, Comput. Methods Appl. Mech. Engrg. 195 (2006) 40364049 .

[23] G. Rapin, G. Lube, A stabilized three-field formulation for advection-diffusion equations, Computing 73 (2004) 155-178.

[24] A. Russo, Bubble stabilization of finite element methods for the linearized incompressible Navier-Stokes equations, Comput. Methods Appl. Mech. Engrg. 132 (1996) 335-343.

[25] P.J. Silvester, N. Kechkar, Stabilised bilinear-constant velocity-pressure finite elements for the conjugate gradient solution of the Stokes problem, Comput. Methods Appl. Mech. Engrg. 79 (1990) 71-86. 